\title{
MAXIMA: A balloon-borne cosmic microwave background anisotropy experiment
}

B. Rabii ${ }^{\text {a) }}$ and C. D. Winant ${ }^{\text {b) }}$

Department of Physics, University of California, Berkeley, California 94720; Space Sciences Laboratory, University of California, Berkeley, California 94720; and Center for Particle Astrophysics,

University of California, Berkeley, California 94720

J. S. Collins

Department of Physics, University of California, Berkeley, California 94720 and Space Sciences Laboratory, University of California, Berkeley, California 94720

A. T. Lee

Department of Physics, University of California, Berkeley, California 94720; Space Sciences Laboratory, University of California, Berkeley, California 94720; Center for Particle Astrophysics,

University of California, Berkeley, California 94720; and Physics Division,

Lawrence Berkeley National Laboratory, Berkeley, California 94720

P. L. Richards

Department of Physics, University of California, Berkeley, California 94720; Space Sciences Laboratory, University of California, Berkeley, California 94720; and Center for Particle Astrophysics,

University of California, Berkeley, California 94720

M. E. Abroe

School of Physics and Astronomy, University of Minnesota, Twin Cities, Minneapolis, Minnesota 55455

S. Hanany

School of Physics and Astronomy, University of Minnesota, Twin Cities, Minneapolis, Minnesota 55455

and Center for Particle Astrophysics, University of California, Berkeley, California 94720

B. R. Johnson

School of Physics and Astronomy, University of Minnesota, Twin Cities, Minneapolis, Minnesota 55455

P. Ade

Physics Department, Cardiff University, Cardiff CF24 3 YB, United Kingdom

A. Balbi

Universita' di Roma Tor Vergata, 00133 Roma, Italy

J. J. Bock

Jet Propulsion Laboratory, Pasadena, California 91109 and California Institute of Technology, Pasadena, California 91125

J. Borrill and R. Stompor

Computational Research Division, Lawrence Berkeley National Laboratory, Berkeley, California 94720

and Space Sciences Laboratory, University of California, Berkeley California 94720

A. Boscaleri and E. Pascale

IFAC-CNR, 50127 Firenze, Italy

P. de Bernardis

Universitá di Roma La Sapienza, I-00185 Roma, Italy

P. G. Ferreira

Astrophysics and Theoretical Physics, University of Oxford, Oxford OXI 3RH, United Kingdom and Center for Particle Astrophysics, University of California, Berkeley, California 94720

V. V. Hristov and A. E. Lange

California Institute of Technology, Pasadena, California 91125

A. H. Jaffe

Imperial College, London SW7 2BW, United Kingdom and Center for Particle Astrophysics,

University of California, Berkeley, California 94720

C. B. Netterfield

Physics Department, University of Toronto, Toronto, Ontario M5S 3H8, Canada

G. F. Smoot

Physics Division, Lawrence Berkeley National Laboratory, Berkeley, California 94720

and Department of Physics, University of California, Berkeley, California 94720 
J. H. P. Wu

Department of Physics, National Taiwan University, Taipei 106, Taiwan and Center for Particle Astrophysics, University of California, Berkeley, California 94720

(Received 3 March 2004; accepted 3 June 2006; published online 21 July 2006)

We describe the Millimeter wave Anisotropy eXperiment IMaging Array (MAXIMA), a balloon-borne experiment which measured the temperature anisotropy of the cosmic microwave background $(\mathrm{CMB})$ on angular scales of $10^{\prime}$ to $5^{\circ}$. MAXIMA mapped the CMB using 16 bolometric detectors in spectral bands centered at 150,240 , and $410 \mathrm{GHz}$, with $10^{\prime}$ resolution at all frequencies. The combined receiver sensitivity to CMB anisotropy was $\sim 40 \mu \mathrm{K} \sqrt{\mathrm{s}}$. The bolometric detectors, which were cooled to $100 \mathrm{mK}$, were a prototype of the detectors which will be used on the Planck Surveyor Satellite of the European Space Agency. Systematic parasitic contributions were controlled by using four uncorrelated spatial modulations, thorough cross-linking, multiple independent CMB observations, heavily baffled optics, and strong spectral discrimination. Pointing reconstruction was accurate to $1^{\prime}$, and absolute calibration was better than $4 \%$. Two MAXIMA flights with more than $8.5 \mathrm{~h}$ of CMB observations have mapped a total of $300 \mathrm{deg}^{2}$ of the sky in regions of negligible known foreground emission. MAXIMA results have been released in previous publications and shown to be consistent with the Wilkinson Microwave Anisotropy Probe. MAXIMA I maps, power spectra, and correlation matrices are publicly available at http://cosmology.berkeley.edu/maxima.

(C) 2006 American Institute of Physics. [DOI: 10.1063/1.2219723]

\section{INTRODUCTION}

Millimeter wave Anisotropy eXperiment IMaging Array (MAXIMA) was a balloon-borne experiment designed to measure the anisotropy of the cosmic microwave background $(\mathrm{CMB})$ over a wide range of angular scales $\left(12^{\prime}\right.$ to $\left.5^{\circ}\right)$. Observations were made of $300 \mathrm{deg}^{2}$ of the sky over the course of two flights, in 1998 and 1999. Results have been released ${ }^{1,2}$ and cosmological implications have been explored with the MAXIMA data alone $e^{3-6}$ and combined with other data sets. ${ }^{7-10}$ MAXIMA data have been correlated with those from the Wilkinson Microwave Anisotropy Probe (WMAP). ${ }^{11,12}$ Cross correlations of the MAXIMA I and MAXIMA II data with the map of the same region of the sky from WMAP show that the three experiments detected the same features and that the contributions from uncorrected systematic errors were negligible.

This article, which is derived in part from two Ph.D. dissertations, ${ }^{13,14}$ is an overview of the experimental design and achieved performance of MAXIMA. Section II describes the two MAXIMA science flights. Section III describes the optics and optical characterization. Section IV describes the detector system. Section V describes the cryogenic receiver and support electronics. Section VI discusses the in-flight responsivity calibration. Section VII presents the pointing system and attitude reconstruction.

\section{A. Goals}

The primary scientific objectives of MAXIMA were to distinguish between the inflationary paradigm and topological defect models for the evolution of the universe and to provide information about cosmological parameters, particularly the geometry of the universe.

\footnotetext{
${ }^{a)}$ Electronic mail: bahman@physics.berkeley.edu

${ }^{b)}$ Electronic mail: cwinant@bolo.berkeley.edu
}

A key data product for a $\mathrm{CMB}$ temperature anisotropy experiment is the angular power spectrum of temperature fluctuations, typically expressed in terms of $\ell$, the spherical harmonic multipole quantum number, and $C_{\ell}$ the mean squared amplitude of the spherical harmonic coefficients $a_{\ell m}$ at a given $\ell$. The angular scales observed by MAXIMA corresponded to a range of $\ell=35-1000$ with a resolution of 75 . This range was suited for measurement of the first three acoustic peaks of adiabatic inflationary models. Measurements in this region have been a powerful tool for testing the general predictions of inflation and for parameter estimation. A number of experiments have published significant measurements of this type. ${ }^{11,15}$

MAXIMA data have also been used to test analysis methods and tools. Treatments have been developed for problems such as beam asymmetry, ${ }^{16}$ foreground discrimination, ${ }^{17}$ scan synchronous noise, ${ }^{18}$ and detection of spatial non-Gaussianity. ${ }^{19-21}$

MAXIMA has been used to test new technologies. In particular, MAXIMA was the first CMB experiment to have used $\sim 100 \mathrm{mK}$ spider-web bolometers, similar to those planned for the Planck Surveyor. The combination of these detectors and an adiabatic demagnetization_cooling system provided receiver sensitivity of $\sim 40 \mu \mathrm{K} \sqrt{\mathrm{s}}$ to CMB temperature variations, calculated as the inverse of the quadrature sum of the inverses of the individual detector sensitivities.

\section{B. Cosmological implications}

The primary scientific result from MAXIMA was the angular power spectrum shown in Fig. $1 .^{2}$ This power spectrum leads to a $95 \%$ confidence constraint on the total density of the universe, $\Omega=1.0_{-0.30}^{+0.15}$ in units of the critical density, the physical density of baryons, $\Omega_{b} h^{2}=0.03 \pm 0.01$, the physical density of cold dark matter, $\Omega_{\mathrm{cdm}} h^{2}=0.2_{-0.1}^{+0.2}$, and the 


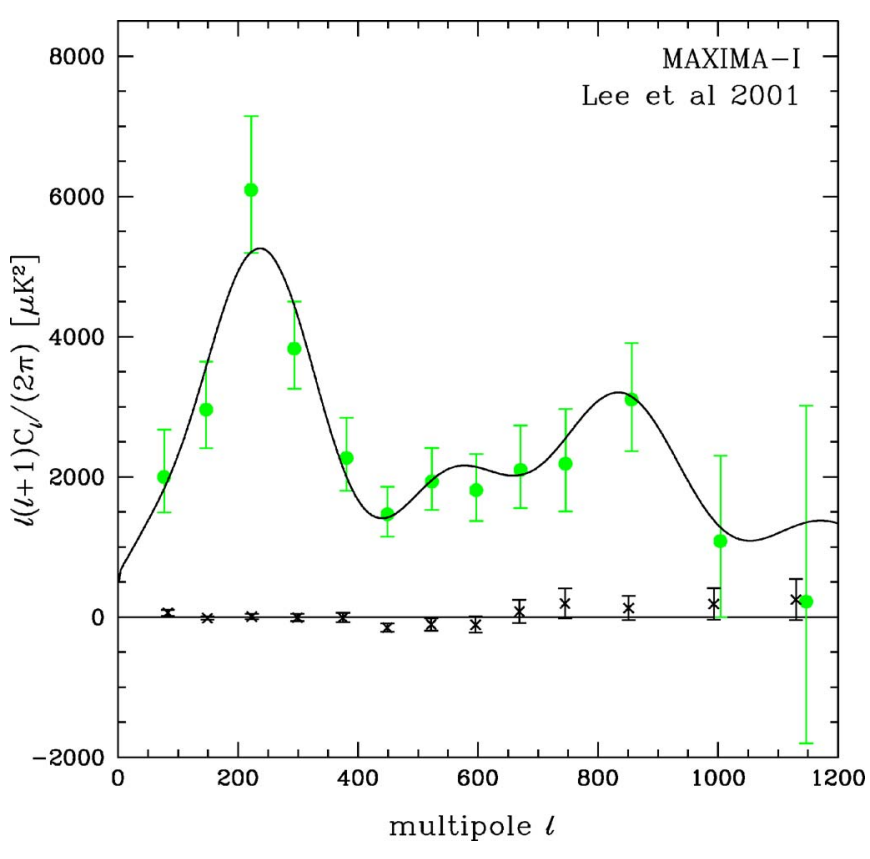

FIG. 1. (Color online) The MAXIMA power spectrum of the CMB computed using a hybrid analysis of $5^{\prime}$ resolution (up to $\ell=335$ ) and $3^{\prime}$ resolution (over $\ell=335$ ) maps. $\ell$ is the spherical harmonic multipole principal quantum number. $C_{\ell}$ is the power spectrum of angular fluctuations. $\ell(\ell$ $+1) C_{\ell} / 4 \pi$ is the total power per logarithmic interval in $\ell$. The first peak in the power spectrum is a powerful probe of the total energy density of the universe (Refs. 3-6). Error bars show one standard deviation statistical uncertainties. The solid curve is the power spectrum of the best fit model from Ref. 5 with $\Omega_{b}=0.1, \Omega_{\mathrm{cdm}}=0.6, \Omega_{\Lambda}=0.3, n=1.08$, and $h=0.53$. The crosses are the power spectrum of the difference between the map from one detector and the combined map from the other two detectors used for the $3^{\prime}$ analysis (Ref. 2) It is consistent with zero.

spectrum of primordial scalar fluctuations, $n_{s}=1.08 \pm 0.1 .^{5}$ All of these results are within one standard deviation of the presently accepted values. ${ }^{11}$

This article is focused on experimental techniques; cosmological interpretation of results is not discussed further.

\section{Technical overview}

MAXIMA was a bolometric instrument which measured CMB temperature fluctuations in frequency bands centered at 150,240 , and $410 \mathrm{GHz}$. In order to reduce the effects of atmospheric emission, observations were made from an altitude of $\sim 40 \mathrm{~km}$ during multiple balloon flights. The relatively short duration of the balloon-borne observations was offset by the use of a 16 element array of single color photometers with extremely sensitive detectors. The telescope was an off-axis Gregorian system with a $1.3 \mathrm{~m}$ diameter primary mirror providing a $10^{\prime}$ beam size [full width at half maximum (FWHM)] for all detectors. The combination of this angular resolution and $\sim 100 \mathrm{deg}^{2}$ of sky coverage made the experiment sensitive over a wide range of angular scales. The scan pattern was compact and well cross-linked. The use of three spectral bands allowed discrimination between the CMB and foreground sources. MAXIMA benefited from precise pointing reconstruction ( $\left.1^{\prime} \mathrm{rms}\right)$ and accurate calibration (4\%). The instrument was designed to survive repeated balloon flights and was successfully recovered after two

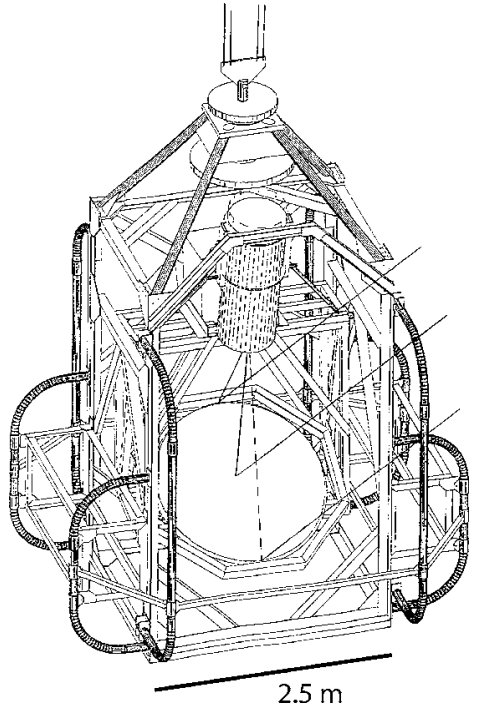

FIG. 2. A drawing of the MAXIMA telescope from an elevated front/side perspective. Rays representing the telescope beam are shown reflecting from the primary mirror into the cryogenic receiver. Electronics housed in the rectangular boxes on the sides of the instrument include the pointing system, data multiplexers and digitizers, and telemetry and command interfaces. Near the top of the telescope were motors controlling azimuthal orientation. The inner frame consisting of the primary mirror and the receiver was tilted relative to the outer frame to change the telescope elevation. The gondola frame was covered in lightweight aluminum-covered builders foam (not shown) and the primary mirror is surrounded by a scoop built of aluminum sheet (not shown), both of which shielded the telescope and receiver from stray optical and radio frequency radiation.

MAXIMA science flights and an instrument testing flight. A drawing of the MAXIMA telescope is shown in Fig. 2.

\section{OBSERVATIONS}

The first science flight, MAXIMA I, was launched on 2 August 1998 at 00:58 UT (1 August 1998, $19: 58$ local time) from the National Scientific Balloon Facility (NSBF) in Palestine, TX (latitude $31.8^{\circ} \mathrm{N}$, longitude $95.7^{\circ} \mathrm{W}$ ). The maximum float altitude of $37.5 \mathrm{~km}$ was reached at 4:35 UT. The telescope traveled $189 \mathrm{~km}$ west and less than $1 \mathrm{~km}$ south before reaching maximum altitude. At float, the telescope drifted $405 \mathrm{~km}$ west and less than $1 \mathrm{~km}$ north. Descent began $3.8 \mathrm{~h}$ later at 8:22 UT. Summer flights from the NSBF in Palestine were limited to a range of approximately $600 \mathrm{~km}$. The MAXIMA I flight was relatively short due to fast highaltitude winds.

Four observations were conducted during the flight. First, the CMB dipole was observed in order to calibrate the responsivity of the detectors. The dipole observation was started before reaching float altitude and lasted from 03:37 to 04:11 UT. Next, two overlapping, cross-linked scans of CMB anisotropy were conducted over a $122 \mathrm{deg}^{2}$ region in the vicinity of the Draconis constellation. These scans occurred from 04:21 to 05:59 UT and from 06:02 to 07:24 UT. Finally, observations were made of Jupiter to characterize the telescope beams and to calibrate the $410 \mathrm{GHz}$ detectors, which were insensitive to the CMB dipole. Jupiter was observed from 07:30 until 08:04 UT.

The Sun was at least $20^{\circ}$ below the horizon for all observations. The Moon was below $20^{\circ}$ elevation during $\mathrm{CMB}$ 


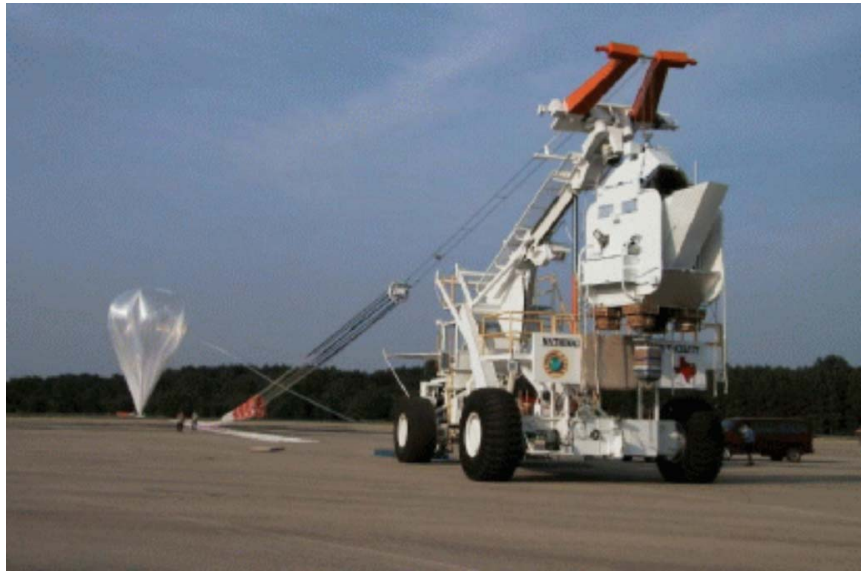

FIG. 3. (Color online) The MAXIMA telescope hanging from the launch vehicle shortly before the MAXIMA II flight on 16 June 1999.

observations and below the horizon for over an hour of the second observation. While above the horizon, it was at least $70^{\circ}$ from the scan region. The relative position of the Moon differed by $20^{\circ}$ azimuth and $10^{\circ}$ elevation between the two CMB scans. During the dipole observation, the Moon was at $30^{\circ}$ elevation, $20^{\circ}$ below the scan. The Moon was below the horizon during the Jupiter scan. The Moon was $68 \%$ full during the flight.

The instrument was launched a second time (MAXIMA II) on 17 June 1998 at 00:07 UT (16 June 1999, 19:07 local time). Figure 3 is a photograph taken shortly before the launch. The telescope traveled $42 \mathrm{~km}$ east and $9 \mathrm{~km}$ south before reversing direction and reaching maximum altitude at a position $97 \mathrm{~km}$ west and $1 \mathrm{~km}$ south of the launch. The maximum float altitude of $38.0 \mathrm{~km}$ was reached at 04:34 UT. At float, the telescope drifted $490 \mathrm{~km}$ west and $42 \mathrm{~km}$ north. Descent began $7.8 \mathrm{~h}$ later at 12:21 UT. The relatively slow high-altitude winds of early summer allowed us a considerably longer flight than MAXIMA I.

As with MAXIMA-I, two CMB observations and two calibration scans were conducted. The first was an observation of Mars from 03:14 to 03:52 UT. After that, approximately $1 \mathrm{~h}$ was spent on maintenance of the receiver cryogenic systems. Two overlapping, cross-linked CMB scans were conducted from 05:04 to 07:29 UT and from 07:31 to 09:40 UT. The observed region had an area of $225 \mathrm{deg}^{2}$ and overlapped the MAXIMA-I region by $50 \mathrm{deg}^{2}$. A calibration scan of the CMB dipole was conducted from 09:42 to 10:19 UT. Further data were recorded from 10:20 to 11:59 UT as a test of the daytime performance of the instrument.

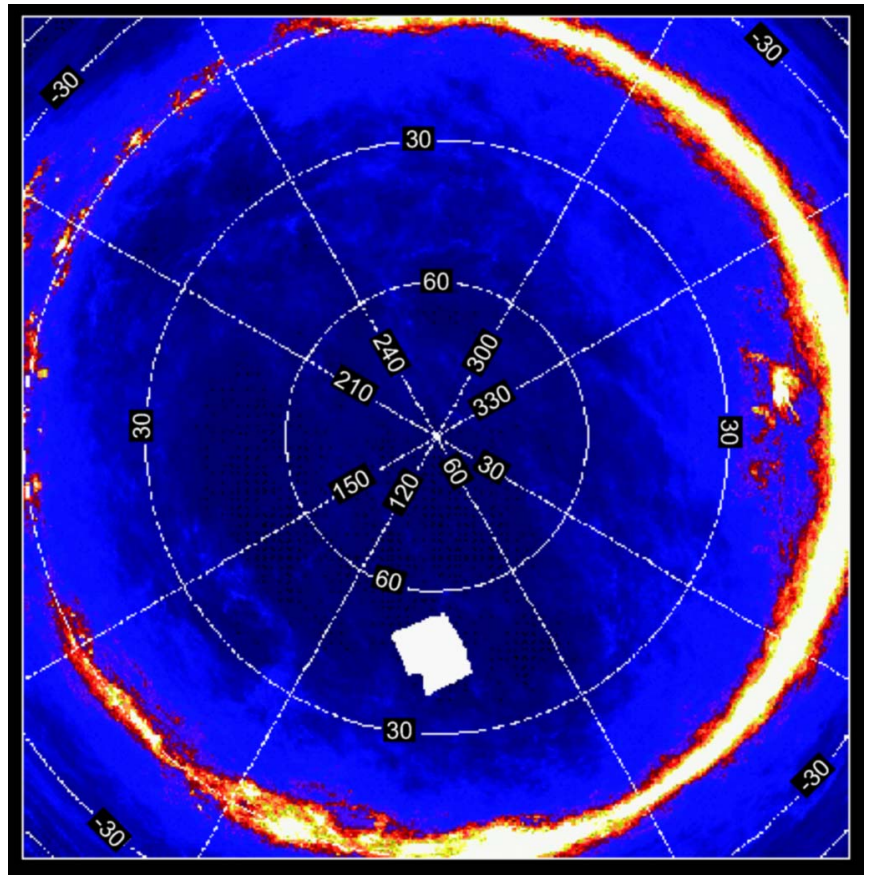

FIG. 4. (Color online) The MAXIMA I CMB observation scan (white) is plotted over emissions from galactic dust taken from the Berkeley-Durham IRAS-Dirbe map of the northern galactic hemisphere dust emission extrapolated to $150 \mathrm{GHz}$ (Ref. 46). Areas of the sky with low dust emission appear as dark regions of the map. The MAXIMA I CMB observation region is constrained to $b>30^{\circ}$.

The Sun rose to $-20^{\circ}$ elevation at 09:24 UT and to $0^{\circ}$ elevation at 11:17 UT. Data collected after 10:20 UT have been used only as test data for future daytime balloon flights. The Moon was $17 \%$ full during the flight and was below the horizon during the dipole observation and both $\mathrm{CMB}$ observations. During the Mars observation, the Moon was $75^{\circ}$ from the scan.

The scans for both flights are summarized in Table I. The observations of MAXIPOL, the follow-up experiment to MAXIMA, are discussed in Ref. 22.

\section{A. Sky selection}

The main constraints on sky selection were celestial foregrounds. The scan regions had a predicted dust temperature anisotropy of $\sim 10.0 \mu \mathrm{K}$ at $150 \mathrm{GHz}$ with rms fluctuations of $\sim 2.5 \mu \mathrm{K}$ in units of equivalent $\mathrm{CMB}$ temperature fluctuation. ${ }^{23}$ Tests of the spectral and angular profiles of the observed signals, as well as cross correlations with known dust maps, confirmed the absence of significant dust contamination in our CMB data. ${ }^{17}$ The MAXIMA I scan region

TABLE I. Flight statistics.

\begin{tabular}{ccccccc}
\hline \hline Flight & $\begin{array}{c}\text { Hours at } \\
\text { maximum } \\
\text { altitude }\end{array}$ & $\begin{array}{c}\text { First CMB } \\
\text { scan } \\
(\mathrm{h})\end{array}$ & $\begin{array}{c}\text { Second CMB } \\
\text { scan } \\
(\mathrm{h})\end{array}$ & $\begin{array}{c}\text { CMB } \\
\text { dipole } \\
(\mathrm{h})\end{array}$ & $\begin{array}{c}\text { Planet } \\
\text { scan } \\
(\mathrm{h})\end{array}$ & $\begin{array}{c}\text { Daytime } \\
\text { test data } \\
(\mathrm{h})\end{array}$ \\
\hline MAXIMA I & $3.78^{\mathrm{a}}$ & 1.63 & 1.37 & 0.57 & 0.57 & 0.00 \\
MAXIMA II & 7.78 & 2.42 & 2.15 & 0.62 & 0.63 & 1.65 \\
\hline \hline
\end{tabular}

${ }^{\mathrm{a}}$ Some calibration data were collected before the telescope reached maximum altitude, resulting in a total scan time greater than the time at maximum altitude. 


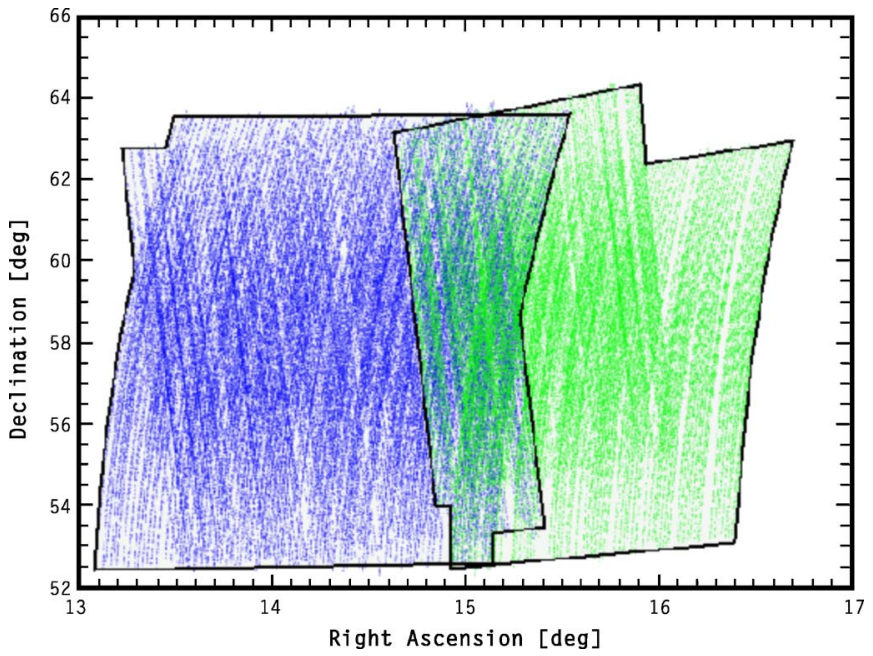

FIG. 5. (Color online) The reconstructed pointing for a single detector in both MAXIMA flights. MAXIMA I is the lighter region on the right, and MAXIMA II is the darker region on the left. The scan region for each flight is boxed, and the $\sim 50 \mathrm{deg}^{2}$ overlap region can be seen at right ascension $\sim 15 \mathrm{~h}$.

was chosen to contain no detectable point sources. For MAXIMA II, this requirement was relaxed so that a few bright sources might be detectable in the anisotropy maps, particularly at $410 \mathrm{GHz}$. No point source contribution was expected from radio or infrared point sources ${ }^{24}$ or from bremsstrahlung or synchrotron radiation ${ }^{25}$ in the 150 and $240 \mathrm{GHz}$ bands in either scan region. The MAXIMA I scan region is shown overlaid with the IRAS-Dirbe dust map in Fig. 4.

The scan regions for the two flights were chosen to have a modest $\left(\sim 50 \mathrm{deg}^{2}\right)$ overlap, both as a consistency check and to facilitate the combination of the data sets. The combined scan pattern is shown in Fig. 5.

\section{OPTICS}

In order to achieve the desired sensitivity to CMB temperature fluctuations up to multipoles of $\ell \simeq 1000$, the telescope had an angular resolution of $10^{\prime}$ FWHM. We used a heavily baffled optical system to reject sidelobe signals from sources such as the Earth, the Moon, and the balloon. Optical background loading was on order of that from the CMB itself. Spectral bands were selected to distinguish CMB from atmosphere and celestial foregrounds.

\section{A. Optical design \\ 1. The telescope}

The MAXIMA telescope was an off-axis Gregorian system, consisting of an $\sim 1.3 \mathrm{~m}$ diameter underfilled primary mirror with cooled, baffled ellipsoidal secondary and tertiary mirrors. Figure 6 shows the optical system and Table II summarizes its optical properties.

The primary mirror was the intersection of a cone and a paraboloid. The cone had an opening angle of $52^{\circ}$. Its apex was at the focus of the parabola, aligned at an angle of $38^{\circ}$ from the axis of the parabola. The focal length from the center of the mirror to the primary focus was $134 \mathrm{~cm}$. The reflecting surface was made of $5000 \AA$ of sputtered aluminum and a protective layer of $2000 \AA$ of $\mathrm{SiO}_{2}$. The emissivity of samples of the surface near $150 \mathrm{GHz}$ was measured to be between $0.27 \%$ and $0.6 \% .{ }^{26}$ Contour measurements gave rms surface accuraces of $8.3 \mu \mathrm{m}$ with fixed focus position and $8.1 \mu \mathrm{m}$ with a shift of focus of $0.61 \mathrm{~mm}$. The $\sim 11 \mathrm{~kg}$

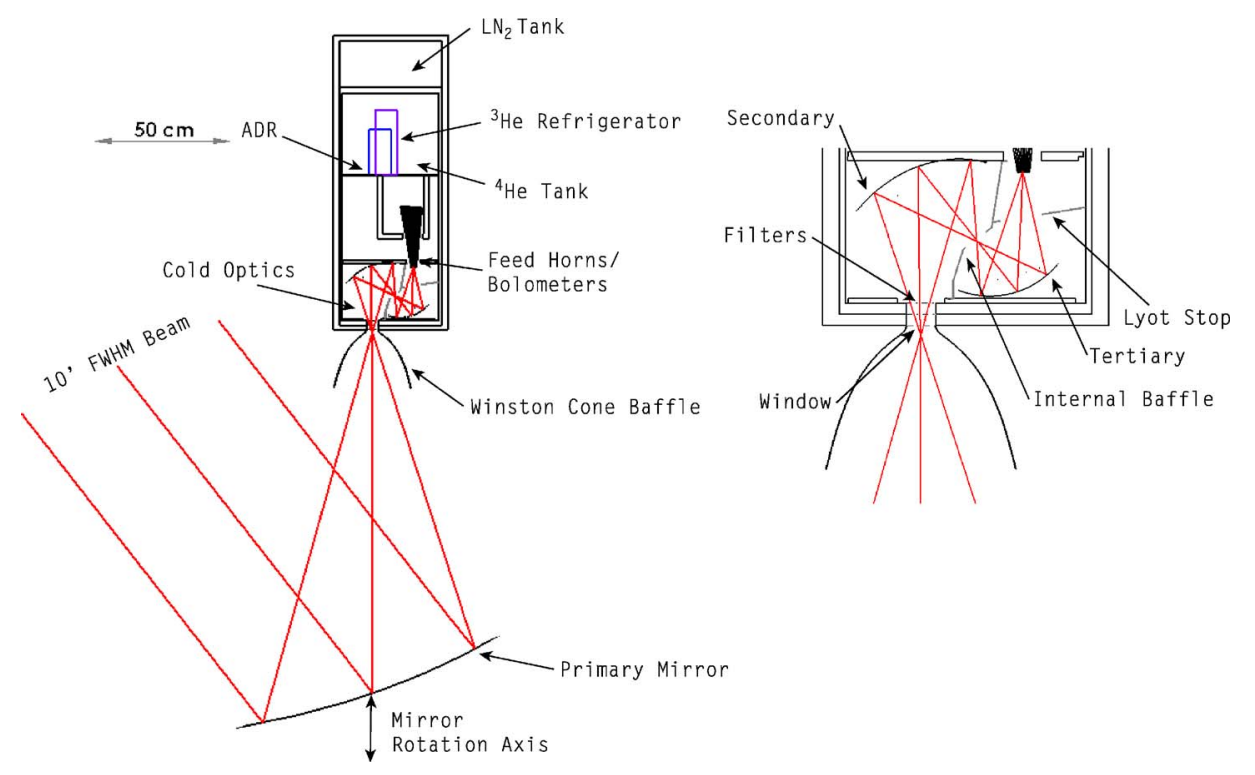

FIG. 6. (Color online) Left: The telescope was a fast $(f / 1)$ Gregorian system. The primary mirror, a $1.3 \mathrm{~m}$ diameter underilluminated paraboloid, was modulated about the indicated axis. A Winston cone baffle (shown outside the cryostat window) blocked radiation not arriving from the primary mirror. Cooled secondary and tertiary mirrors corrected aberrations from the primary and reimaged the focal plane on an array of feedhorns which channeled light to the bolometers. The upper portion of the cryostat housed the cooling systems. Right: The focal plane was baffled from stray light in several ways. At least five edge diffractions with angles of up to $65^{\circ}$ were required for rays outside of the defined throughput to arrive to the focal plane. The internal baffles were blackened with millimeter wave absorptive material and the Winston cone baffle restricted the throughput to radiation from the primary mirror. A Lyot stop defined the illumination on the primary mirror. Filters which defined the measurement bands were located at the prime focus near the cryostat window, at the Lyot stop, and after the feedhorns. 
TABLE II. Optical parameters of the MAXIMA telescope.

\begin{tabular}{|c|c|}
\hline \multicolumn{2}{|c|}{ System parameters } \\
\hline Effective pupil diameter & $835 \mathrm{~mm}$ \\
\hline System focal length & $1521 \mathrm{~mm}$ \\
\hline Focal ratio & 1.8 \\
\hline Plate scale & $0.4 \mathrm{deg} \mathrm{cm}^{-1}$ \\
\hline \multicolumn{2}{|c|}{ Primary mirror } \\
\hline Dimensions & $1328 \times 1267 \mathrm{~mm}$ \\
\hline Conic constant $^{\mathrm{a}}$ & -1 \\
\hline Vertex radius of curvature & $2400 \mathrm{~mm}$ \\
\hline Focal length & $1340 \mathrm{~mm}$ \\
\hline \multicolumn{2}{|c|}{ Secondary mirror } \\
\hline Radius & $210 \mathrm{~mm}$ \\
\hline Conic constant & -0.126 \\
\hline Vertex radius of curvature & $168 \mathrm{~mm}$ \\
\hline \multirow[t]{4}{*}{ Aspheric coefficients ${ }^{\mathrm{a}}$} & $A=2.15 \times 10^{-5}$ \\
\hline & $B=-9.07 \times 10^{-8}$ \\
\hline & $C=1.55 \times 10^{-10}$ \\
\hline & Tertiary mirror \\
\hline Radius & $180 \mathrm{~mm}$ \\
\hline Conic constant & -0.227 \\
\hline Vertex radius of curvature & $112.5 \mathrm{~mm}$ \\
\hline \multirow[t]{3}{*}{ Aspheric coefficients } & $A=6.59 \times 10^{-5}$ \\
\hline & $B=-5.03 \times 10^{-7}$ \\
\hline & $C=1.58 \times 10^{-9}$ \\
\hline
\end{tabular}

${ }^{\mathrm{a}}$ Surface parameters are given in terms of $z=c r^{2} /\left[1+\sqrt{1-(1+k) c^{2} r^{2}}\right]+A r^{4}$ $+B r^{6}+C r^{8}$, where $z$ is the sag of the surface, $c$ is the curvature at the vertex, $k$ is the conic constant, and $r$ is the perpendicular distance from the symmetry axis.

mirror was constructed by Dornier Satellitensysteme (Germany) from a lightweight graphite-epoxy honeycomb to facilitate this modulation. During observations the primary mirror was modulated by rotating around the axis connecting its center and the primary focus. The modulation changed the telescope beam illumination of the mirror by less than $0.4 \%$.

The design of the reimaging system was driven by the fixed optical parameters of the primary mirror, a need for a cooled aperture stop (Lyot stop) and a requirement for a diffraction limited field of view of $1 \mathrm{deg}^{2}$ at frequencies lower than about $410 \mathrm{GHz}$. It was also preferable to cool the secondary optics to reduce the optical load on the detectors, because emission from the telescope was expected to be a dominant source of detector noise at 150 and $240 \mathrm{GHz}$, given the expected $\sim 10 \mathrm{nV} \mathrm{Hz}^{-0.5}$ amplifier noise, see Table III (Ref. 27) and Sec. IV B. Cooling the optics required a

TABLE III. Predicted optical load during flight.

\begin{tabular}{cccc}
\hline \hline Source & $P_{150}(\mathrm{pW})$ & $P_{240}(\mathrm{pW})$ & $P_{410}(\mathrm{pW})$ \\
\hline CMB & 0.11 & 0.10 & 0.01 \\
Primary mirror $^{\mathrm{a}}$ & 0.18 & 0.39 & 0.64 \\
Atmosphere $^{\mathrm{b}}$ & $<0.01$ & 0.03 & 0.05 \\
Cold mirrors $^{\mathrm{c}}$ & 0.10 & 0.10 & 0.01 \\
Total & 0.39 & 0.52 & 0.71 \\
\hline \hline
\end{tabular}

$\overline{{ }^{a} \text { Assumed primary mirror emissivities of } 0.5 \%, 0.6 \% \text {, and } 0.8 \% \text { for the } 150 \text {, }}$ 240, and $410 \mathrm{GHz}$ bands, respectively.

${ }^{\mathrm{b}}$ Atmospheric load was modeled with ATM (Ref. 27).

${ }^{\mathrm{c}}$ Approximation (factor of $\sim 2$ ) for polished aluminum. compact system.

The secondary optics consisted of ellipsoidal secondary and tertiary mirrors that had aspheric corrections and respective diameters of 21 and $18 \mathrm{~cm}$. They were diamond turned from solid aluminum which gave an optical quality surface at visible wavelengths. The back of each mirror was lightweighted to reduce the mass and heat capacity. The mirrors were manufactured and assembled inside a baffled optics box by Speedring Systems (U.S.A.). Speedring Systems also used laser interferometry to verify the alignment and optical performance of the secondary optics. The optics box was housed within the MAXIMA cryostat and was maintained at $\sim 4 \mathrm{~K}$ by liquid helium. The interior baffles of the box were blackened with an $\sim 0.5 \mathrm{~cm}$ thick layer of combined Stycast 2850 FT black epoxy [Emerson \& Cuming Microwave Products (U.S.A.)], carbon lampblack, and $175 \mu \mathrm{m}$ diameter glass beads, which had been demonstrated to be an effective far-infrared absorber. ${ }^{28}$

We used CODE V, a software package by Optical Research Associates (U.S.A.), to design the optical system and assess its performance. The telescope provided beams with full width at half maximum of $10^{\prime}$ at all frequencies; at 150, 240, and $410 \mathrm{GHz}$ the worst Strehl ratios (wave front errors) over the entire focal plane were 0.97 (0.03), 0.94 (0.04), and 0.83 (0.07), respectively. [Strehl ratios (wave front errors) larger (smaller) than $0.82(0.06)$ were considered within the diffraction limit.] The focal surface was curved for which we accounted with the placement of the feedhorns. Figure 7 shows a schematic view of the focal plane from the point of view of the detectors.

We chose a Gregorian system with three foci because it could be well baffled, see Fig. 6. A large Winston cone baffle outside the cryostat window was designed to admit throughput only from the primary mirror. A ray arriving from a direction outside the primary mirror required five edge diffractions with angles as large as $65^{\circ}$ to arrive at the focal plane.

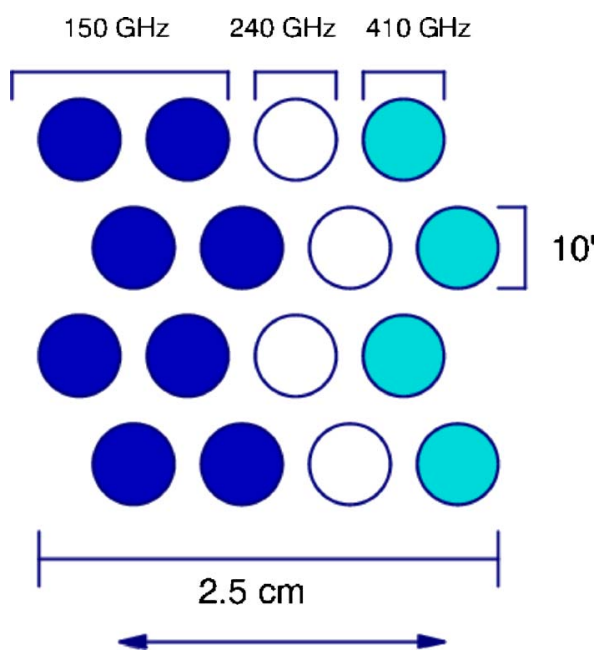

FIG. 7. (Color online) The layout of the MAXIMA focal plane as viewed from behind the bolometers. The arrows indicate the scan direction for azimuthal modulation at constant elevation. All 16 channels project onto the sky with a $10^{\prime}$ FWHM beam size. The width of the focal plane was $2.5 \mathrm{~cm}$. 


\section{Lyot stop}

We located the liquid- ${ }^{4} \mathrm{He}$-cooled Lyot (aperture) stop after the tertiary mirror and before the entrance to the photometers to apodize the illumination of the primary and to terminate the excess field of view at $\sim 4 \mathrm{~K}$. The Lyot stop was fabricated from a $0.63 \mathrm{~cm}$ thick sheet of Eccosorb MF124, a microwave absorber manufactured by Emerson \& Cuming Microwave Products (U.S.A.). This thickness provided adequate absorption of excess radiation. ${ }^{29}$

The Lyot stop had an elliptical opening with semimajor and semiminor axis lengths of 2.04 and $1.75 \mathrm{~cm}$. We achieved a well focused beam despite the thickness of the material by tapering the opening to a knife edge at an angle of $30^{\circ}$. The face pointed towards the sky was covered with a layer of $25 \mu \mathrm{m}$ thick aluminum foil which reflected radiation that would otherwise have been transmitted through the thinnest section of the taper.

\section{Feedhorns}

We used a feedhorn coupled array to shield the bolometers from instrumental optical load and from radio frequency interference (RFI). ${ }^{30}$ The optical signal from the sky was fed to 16 individual photometers through back-to-back circular aperture copper feedhorns. At $150 \mathrm{GHz}, 10^{\prime}$ FWHM beams were at the diffraction limit of the telescope; therefore we used single-moded straight walled feedhorns at $150 \mathrm{GHz}$. The theoretical beam patterns for a single-moded straight walled feedhorn were well understood. ${ }^{31}$ The higher frequency channels were multimoded and had Winston cone feedhorns. ${ }^{32}$ All feedhorns were designed to create $10^{\prime}$ FWHM beams. The back-to-back feedhorn design was used to collimate the radiation, which was useful for spectral filtering.

The opening diameter of the feedhorns was quantified in terms of $N F \lambda$, where $\lambda$ is the wavelength of observation, $F$ is the focal ratio ( $f$ number) at the focal surface opening, and $N$ is a numerical factor. $F$ is determined by the FWHM of the beam and by the reimaging scheme. We optimized the aperture efficiency ${ }^{30}$ by choosing $N \simeq 2[N F \lambda=(6.10: 4.47) \mathrm{mm}$ for the (150:240) $\mathrm{GHz}$ channel feedhorns]. For this value of $N$, the telescope beams were separated by $\sim 20^{\prime}$ on the sky.

The feedhorns were fabricated by machining aluminum mandrils to define the interior surface. The mandrils were then electroplated with copper, and the aluminum was etched away in a bath of $\mathrm{NaOH}$. The exterior surfaces of the feedhorns were then trimmed on a lathe, and the interior surfaces were polished. The feedhorns and bolometer array are shown in Fig. 8.

\section{Frequency bands, filters, and detector backshorts}

MAXIMA observed in frequency bands were centered at 150, 240, and $410 \mathrm{GHz}$. The 150 and $240 \mathrm{GHz}$ bands were primarily used to measure the CMB. The $410 \mathrm{GHz}$ band monitored emission from atmosphere, galactic dust, and extragalactic infrared point sources. Data from the three observation bands were used to discriminate spectrally between various signal sources.

The frequency bands were primarily defined by metalmesh filters located in the collimated beams between the

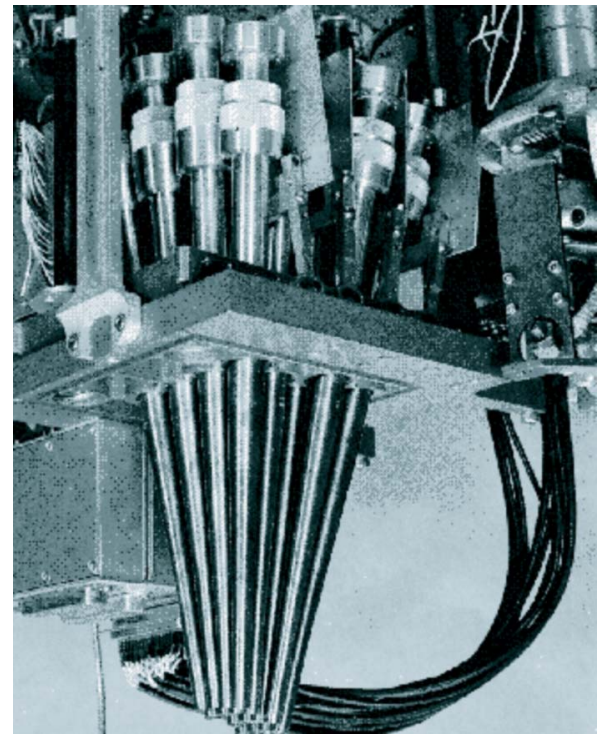

FIG. 8. (Color online) A photograph of the arrays of feedhorns and bolometers. The entrances of the feedhorns at the bottom defined the curved focal surface. The band defining metal-mesh filters and bolometers for each channel were assembled inside aluminum holders at the top. The horn array at $3 \mathrm{~K}$ and the bolometer array at $100 \mathrm{mK}$ were separated by a $0.5 \mathrm{~mm}$ gap. The cold bolometer-filter stage was supported by three thin walled Vespel SP-22 tubes.

feedhorns and the bolometers. The 240 and $410 \mathrm{GHz}$ channels had both high-pass and low-pass metal-mesh filters. The $150 \mathrm{GHz}$ channels only had a low-pass metal-mesh filter; the section of circular waveguide between the back-to-back straight cones acted as a high-pass filter.

The filters were built from thin metallic meshes supported on Mylar film substrates by Ade who was then at Queen Mary Westfield College, London, England. The transmission of the filter was determined by the pattern and geometry of the film, and the number and spacing of the stack of meshes. A thin copper substrate was evaporated onto a taut of $\sim 1.5 \mu \mathrm{m}$ thick Mylar sheet suspended on a stainless steel ring. The metal was patterned via photolithograpy in either a capacitive (low-pass), inductive (high-pass), or resonant (bandpass) geometry. The steepness of the cutoff was improved by stacking multiple meshes separated by $\lambda_{0} / 4$, where $\lambda_{0}$ was the cutoff frequency of the filter. These filters could be repeatedly cryogenically cycled without any stress fractures or significant performance change. They have been demonstrated to have high transmission and sharp cutoffs. ${ }^{33}$

The band defining filters leaked slightly at harmonics of the cutoff frequency. Three low-pass filters between the cryostat window and the entrance to the feedhorns blocked these leaks and provided overall rejection at higher frequencies. Two of these filters were low-pass metal-mesh filters with cutoffs at 480 and $570 \mathrm{GHz}$. The last was an absorptive alkali-halide filter and had a cutoff frequency of $1650 \mathrm{GHz}$.

The $150 \mathrm{GHz}$ bolometers were suspended in resonant optical cavities with a depth of $\lambda_{\text {obs }} / 4$. This depth was set by fixing a flat brass backshort behind each bolometer. For narrow frequency bands, this geometry would establish a standing wave in the feedhorn and bolometer cavity with the bolometer optimally placed at a maximum of the electric field 


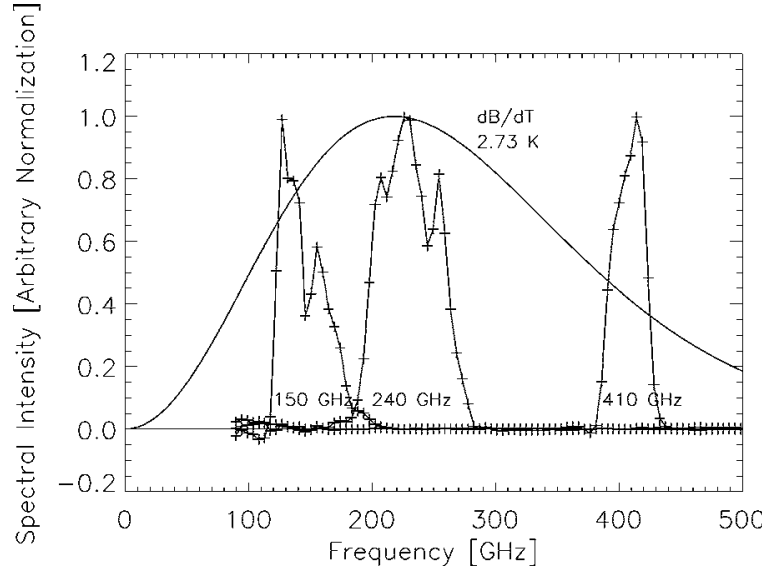

FIG. 9. The measured, normalized spectral response for one detector of each color. These spectra were measured before flight with a Michelson Fourier spectrometer. The solid curve represents the derivative of the emission spectrum of a $2.73 \mathrm{~K}$ blackbody with respect to temperature $d B / d T$.

and would minimize radiation losses incurred by transmission past the bolometer. The results of this strategy are discussed in Sec. III B.

\section{Neutral density filter}

We placed a $1 \%$ transmitting neutral density filter (NDF) at the intermediate focus between the secondary and tertiary mirrors for optical tests with a $300 \mathrm{~K}$ load. The NDF was made by evaporating a thin continuous metal film onto a taut Mylar sheet stretched over a stainless steel ring and was provided by Ade. The NDF was mounted on an aluminum slider which could be manually moved in and out of the beam using a vacuum-sealed linear actuator.

\section{B. Preflight characterization}

We measured the spectral response and optical efficiency of each channel in the laboratory before flight. We measured the beams of the entire telescope and the far sidelobe response of each channel just before flight to verify proper focusing and baffling of the telescope.

\section{Spectral sensitivity}

The transmission spectrum of each channel was measured before MAXIMA I to verify the performance of the band defining filters. These spectral measurements were used to discriminate between foreground and $\mathrm{CMB}$ signals and for calibration from point sources with spectra different from that of the CMB.

We measured the spectral response from $4.8 \mathrm{GHz}$ to $1.2 \mathrm{THz}$, with a resolution of $4.8 \mathrm{GHz}$ using a Michelson Fourier spectrometer. ${ }^{34}$ One such spectrum from each of the three frequency bands is shown in Fig. 9. The noise in the spectrum rose significantly below $90 \mathrm{GHz}$ and above $1 \mathrm{THz}$ due to the reduced efficiency of the beam splitter. We also saw increased noise at $540 \mathrm{GHz}$ due to a strong water absorption line. We estimated statistical errors of $2 \%$ for the $150 \mathrm{GHz}$ bands, $14 \%$ for the $240 \mathrm{GHz}$ bands, and $7 \%$ for the $410 \mathrm{GHz}$ bands.

The spectral response for all photometers of a given frequency band was morphologically similar. The FWHM band edges averaged over each set of detectors were 124-164 GHz (150 GHz nominal), $199-258 \mathrm{GHz}$ (240 GHz nominal), and 393-423 GHz (410 GHz nominal). This gave absolute (fractional) bandwidths of $40 \mathrm{GHz}$ $(27.8 \%)$ at $150 \mathrm{GHz}, 59 \mathrm{GHz}(25.8 \%)$ at $240 \mathrm{GHz}$, and $30 \mathrm{GHz}(7.3 \%)$ at $410 \mathrm{GHz}$. The measured band centers varied by several gigahertz. In the case of the $150 \mathrm{GHz}$ channels, the measured bands were asymmetric.

We checked for high frequency leaks in the photometers before each flight by measuring each bolometer's response to a Rayleigh-Jeans source through a series of roomtemperature high-pass filters. The source consisted of a $77 \mathrm{~K}$ cold load periodically chopped with $300 \mathrm{~K}$ blades. We placed a conservative estimate that $\sim 1 \%$ or less of the chopped optical power detected by each bolometer leaked above the high frequency cutoff of the nominal band for that photometer. This was a stringent test of susceptibility to foregrounds with steeply rising emission spectra over our observation bands $\left(B_{\nu} \propto \nu^{x}, x \geqslant 2\right)$, such as galactic and extragalactic dust, the atmosphere, and albedo.

\section{Optical efficiency}

The optical efficiency of a detector $\epsilon(\nu)$ is defined as the ratio of optical power detected to that which enters the telescope and is a frequency dependent quantity. The total optical power detected $P_{\text {detected }}$ can be expressed as

$$
P_{\text {detected }}=A \Omega \int_{\nu} \epsilon(\nu) I(\nu) d \nu \simeq A \Omega<\epsilon(\nu)>\int_{\nu_{l}}^{\nu_{h}} I(\nu) d \nu
$$

where $A \Omega=0.041 \mathrm{~cm}^{2} \mathrm{sr}$ is the throughput of the telescope and $I(\nu)$ is the spectral intensity of the observed source. The average optical efficiency $\langle\epsilon(\nu)\rangle$ is defined between FWHM frequencies $\nu_{l}$ and $\nu_{h}$.

In the laboratory, we determined the average optical efficiency, $\langle\epsilon(\nu)\rangle$, for each channel over its effective bandwidth. Using the neutral density filter to avoid saturation, we measured the load curve (i.e., bolometer resistance versus applied electrical power) of each bolometer with a $300 \mathrm{~K}$ optical load filling the throughput. We repeated the measurement with a $77 \mathrm{~K}$ load. For points of equal resistance on the two load curves, the bolometer was electrically heated to an equal total (electrical plus optical) power; the difference in electrical power thus gave the difference in detected optical power from 300 to $77 \mathrm{~K}$ loads.

We measured only the optical efficiency of the bolometers and the cold optics, as it was impractical to fill the primary mirror with a calibrated diffuse source. The primary mirror had an absorptivity of order $0.5 \%$ over all observation bands and should not degrade the overall optical efficiency significantly.

We made this series of measurements twice before MAXIMA I. Between measurements, we improved the optical efficiencies of the detectors by polishing the feedhorns and by implementing the $\lambda_{0} / 4$ backshorts described in Sec. III A in all $150 \mathrm{GHz}$ channels. The new backshorts improved 
TABLE IV. Optical efficiencies for the MAXIMA receiver.

\begin{tabular}{ccc}
\hline \hline Channel & $\begin{array}{c}\langle\nu\rangle \\
(\mathrm{Hz})\end{array}$ & $\begin{array}{c}\langle\epsilon(\nu)\rangle \\
(\%)\end{array}$ \\
\hline b14 & 150 & 18.4 \\
b15 & 150 & 8.4 \\
b24 & 150 & 20.2 \\
b25 & 150 & 22.8 \\
b34 & 150 & 14.2 \\
b35 & 150 & $\ldots{ }^{\mathrm{a}}$ \\
b44 & 150 & 16.8 \\
b45 & 150 & 15.0 \\
b13 & 240 & 3.9 \\
b23 & 240 & 13.9 \\
b33 & 240 & 9.6 \\
b43 & 240 & 24.2 \\
b12 & 410 & 3.8 \\
b22 & 410 & 3.9 \\
b32 & 410 & 3.9 \\
b42 & 410 & 5.6 \\
\hline \hline
\end{tabular}

${ }^{\mathrm{a}}$ Nonopertational channel at the time of the measurement.

the optical efficiencies of these channels by a factor of 2-4. The efficiencies achieved for all channels are listed in Table IV.

\section{Focusing}

The telescope was focused by bringing the prime foci of the primary mirror and the secondary optics in the receiver together along a unique optical axis. This was initially done using mechanical alignment tools and visible laser tests. We then measured the two-dimensional beam profiles of each channel in the array by performing a raster scan with the telescope over a 7' FWHM source. The source was a halogen lamp mounted at the focus of a $1 \mathrm{~m}$ diameter $f / 1$ paraboloidal mirror. The source was chopped at $5 \mathrm{~Hz}$ so that signal appeared above the $1 / f$ knee in the bolometer signal bands. Measured profiles were compared to those predicted from simulations of the optical system with various types of defocus. We corrected the focus of the telescope iteratively to minimize beam size and asymmetry. Beam contours for both observations are shown in Fig. 10.

\section{Far sidelobe measurements}

We measured the far sidelobe response of the telescope before flight to verify that sources such as the Earth, the Moon, the balloon, and the gondola would not contaminate our measurements of CMB temperature fluctuations.

We aimed a $150 \mathrm{GHz}, 20 \mathrm{~mW}$ Gunn oscillator at the assembled and baffled payload from a distance of $\sim 20 \mathrm{~m}$. The oscillator was chopped at $7 \mathrm{~Hz}$. These measurements, which were done in the near field because of technical limitations, provided a useful indication of the far field sidelobe response. The sidelobes were measured outdoors to reduce secondary reflections off building walls. Nevertheless, it was likely that we detected some reflected signals, which would have caused the sidelobe response measured on the ground to be larger than the in-flight sidelobe response.

For MAXIMA I, the source was mounted on the top of a $35 \mathrm{~m}$ tall building. For MAXIMA II, the source was mounted at the end of the arm of a "cherry picker" truck. The MAXIMA II method more effectively reduced secondary reflections. We scanned across elevation and azimuth from the beam center yielding two orthogonal, one-dimensional sidelobe maps.

The response of the bolometers was linear for signal changes less than $20 \mathrm{~dB}$. We attenuated the output of the source with both a dial attenuator and with $2.54 \mathrm{~cm}$ thick sheets of plywood to keep the response linear over the $80 \mathrm{~dB}$ range of the measurement.
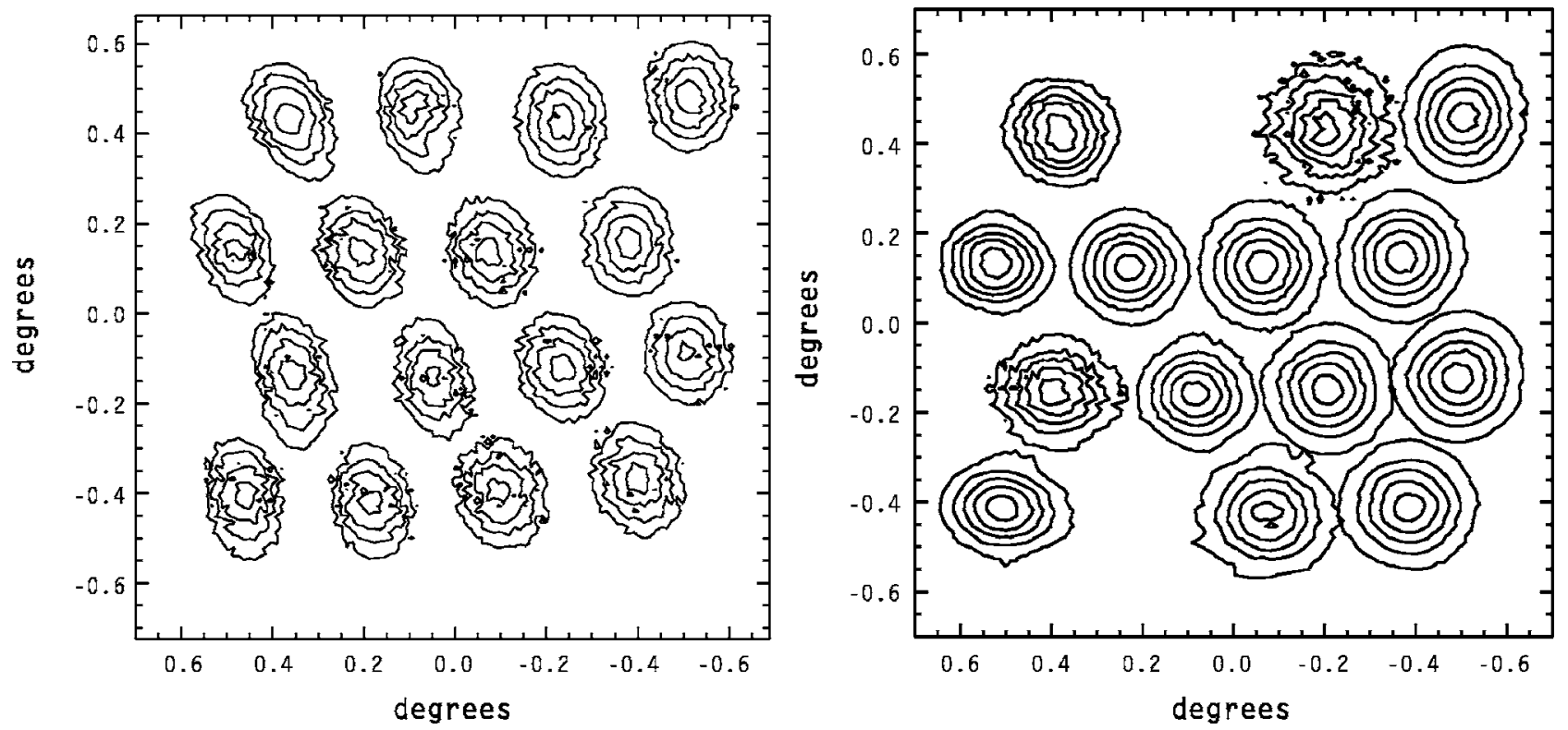

FIG. 10. Beam contour plots for all channels, shown in the reverse of the view in Fig. 7. Left: MAXIMA I: the contours, starting at the center of each beam, represent the 90\%, 70\%, 50\%, and 30\%, levels, respectively. Right: MAXIMA II: The contours represent the 90\%, 70\%, 50\%, 30\%, and 10\%, levels, respectively. Two of the $240 \mathrm{GHz}$ channels were not operational during the MAXIMA II flight. Refinements in focusing techniques between the MAXIMA flights led to improved beam symmetry in MAXIMA II. 

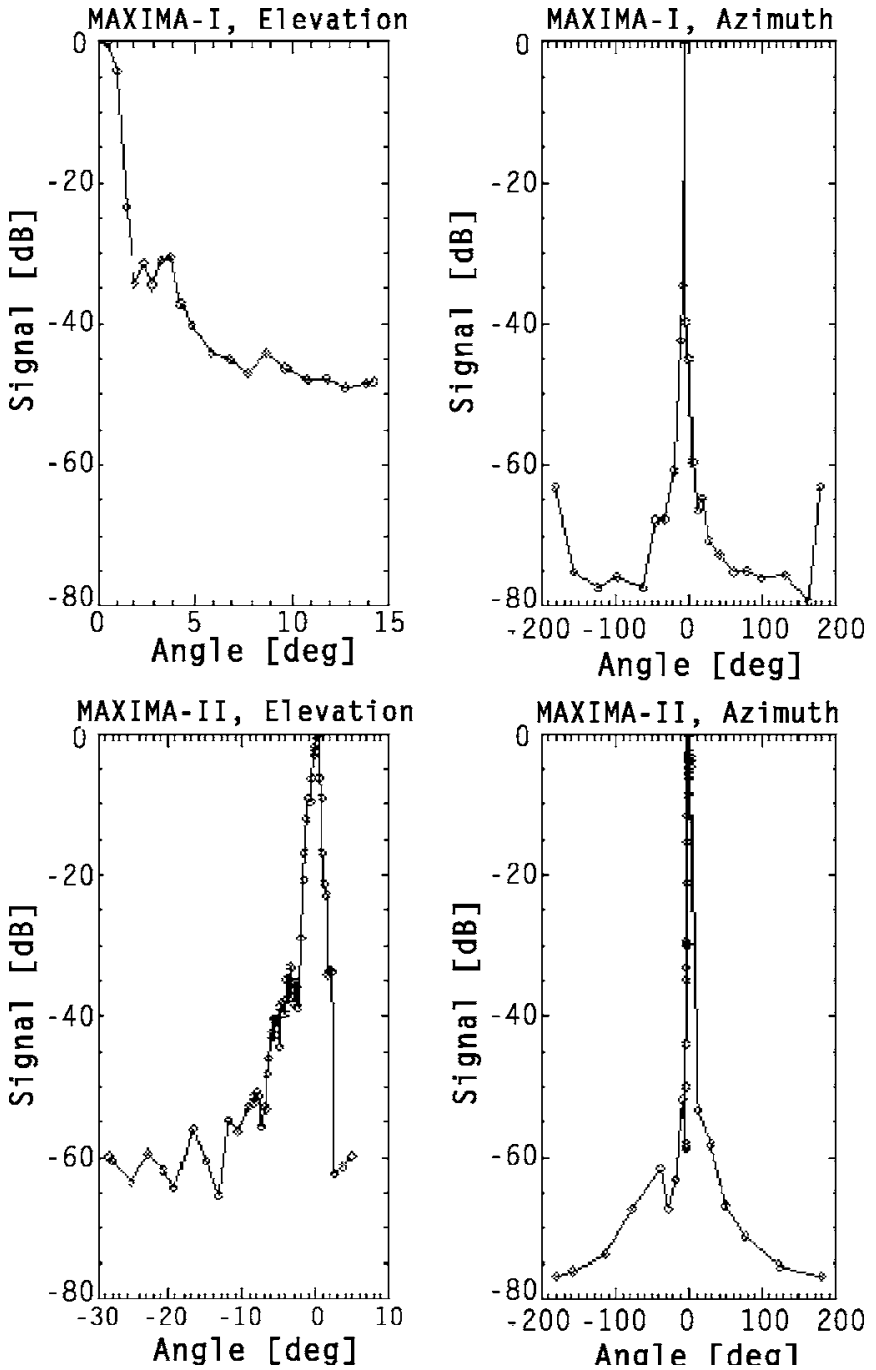

FIG. 11. Data from preflight sidelobe tests. The source was roughly $20 \mathrm{~m}$ from the telescope. Left top: Test data in the elevation direction for MAXIMA I. The angle was that of the telescope above the test source. Right top: Test data in the azimuth direction for MAXIMA I. The telescope was rotated at fixed elevation $\left(\sim 30^{\circ}\right)$. Left Bottom: As above, for MAXIMA II. Most of these data were collected with the source at higher elevation than the telescope beam (negative angles on the $x$ axis). Right bottom: As above, for MAXIMA II, except that the source was moved around the telescope.

The measured far sidelobe response is shown in Fig. 11. The measurement noise floor was roughly $-75 \mathrm{~dB}$. The elevation beam maps had a higher apparent noise floor, probably due to reflected signals. The measured attenuation at $15^{\circ}$ below the beam was sufficient to prevent significant Earthbased sidelobe contamination. The apparent $-60 \mathrm{~dB}$ backlobe in MAXIMA I was suspected to be an artifact of the measurement technique. Even if the backlobe were real, no sources in the nighttime sky would be detectable with this attenuation.

\section{In-flight characterization of beam patterns}

The beam function (i.e., the Legendre polynomial expansion of the beam) was essential for probing CMB power at higher multipoles. We measured the beam patterns of each channel in the array during flight to determine the beam
TABLE V. MAXIMA beams FWHM. Note that $\theta_{\text {maj }}$ and $\theta_{\text {min }}$ represent the major and minor axes. Values were determined from the 50\% contour for each beam. M1 and M2 refer to MAXIMA I and MAXIMA II.

\begin{tabular}{|c|c|c|c|c|c|}
\hline Channel & $\begin{array}{c}\langle\nu\rangle \\
(\mathrm{GHz})\end{array}$ & $\begin{array}{l}\mathrm{M} 1: \theta_{\text {maj }} \\
(\operatorname{arc} \min )\end{array}$ & $\begin{array}{l}\mathrm{M} 1: \theta_{\min } \\
(\operatorname{arc} \min )\end{array}$ & $\begin{array}{l}\text { M2: } \theta_{\text {maj }} \\
(\operatorname{arc} \text { min })\end{array}$ & $\begin{array}{l}\mathrm{M} 2: \theta_{\min } \\
(\operatorname{arc} \min )\end{array}$ \\
\hline b14 & 150 & 11.3 & 9.0 & 10.0 & 9.3 \\
\hline b15 & 150 & 10.8 & 9.0 & 10.1 & 9.1 \\
\hline b24 & 150 & 10.8 & 8.4 & 9.5 & 9.2 \\
\hline b25 & 150 & 10.8 & 8.4 & 9.7 & 9.3 \\
\hline b34 & 150 & 10.8 & 8.4 & 9.5 & 9.5 \\
\hline b35 & 150 & 10.0 & 8.4 & 9.8 & 9.4 \\
\hline b44 & 150 & 10.5 & 9.2 & 10.1 & 9.3 \\
\hline b45 & 150 & 10.8 & 9.0 & 10.0 & 9.4 \\
\hline b13 & 240 & 10.2 & 8.4 & $\ldots^{\mathrm{a}}$ & $\ldots^{\mathrm{a}}$ \\
\hline b23 & 240 & 11.0 & 8.4 & 8.7 & 8.4 \\
\hline b33 & 240 & 11.3 & 7.7 & 8.7 & 8.2 \\
\hline b43 & 240 & 11.5 & 8.4 & $\ldots^{\mathrm{a}}$ & $\ldots^{\mathrm{a}}$ \\
\hline b12 & 410 & 12.0 & 8.3 & 8.8 & 8.1 \\
\hline b22 & 410 & 10.2 & 9.0 & 8.3 & 7.5 \\
\hline b32 & 410 & 13.6 & 7.9 & 8.5 & 8.1 \\
\hline b42 & 410 & 11.0 & 7.7 & 9.5 & 7.4 \\
\hline
\end{tabular}

${ }^{\mathrm{a}}$ Nonoperational channel.

function $B_{\ell}$. Beam measurements were also necessary for point source responsivity calibration.

\section{Measurement}

The beam patterns were measured in flight by observing Jupiter for MAXIMA I and Mars for MAXIMA II. These planets were both small in angular size compared to the beam FWHM and could thus be treated as point sources. They were detected with signal-to-noise ratios of $\sim 100$ for Mars and $\sim 1000$ for Jupiter.

During planet observations, the telescope tracked the planet in azimuth while remaining at fixed elevation. As the planet drifted through the elevation of the observation, the modulation of the primary mirror scanned the beams across the planet many times. As the planet drifted in elevation the spatial response of each beam was measured in two dimensions. The angular offset between the center of the beam and the boresight charge coupled device (CCD) camera was also measured in these scans. The detector data were averaged in fixed azimuth and elevation bins over a prescribed area and resolution through three-point linear interpolation. This beam map was decomposed into the beam function $B_{\ell}$. We measured the beam FWHM from the $50 \%$ contour of the beam map.

We measured the beams for all of the functioning channels during both MAXIMA observations. Two-dimensional beam contours are shown in Fig. 10. The FWHM of the major and minor axes of each beam is listed in Table V.

\section{Symmetry}

The telescope revisited regions of the sky multiple times over the course of a given flight. The scan angles for each observation varied considerably due to sky rotation. In the ideal case where the telescope beams have circular symmetry, the same patch of sky would have filled the beams upon 
TABLE VI. Sources of beam error for one $150 \mathrm{GHz}$ channel.

\begin{tabular}{lc}
\hline \hline \multicolumn{1}{c}{ Source of error } & $\begin{array}{c}\sigma_{\text {FWHM }} \\
(\operatorname{arc~min})\end{array}$ \\
\hline White noise in the detector time stream & 0.09 \\
Bolometer electronic filters & 0.13 \\
Bolometer time constant & 0.06 \\
Phase preserving low-pass filter & 0.14 \\
Pointing reconstruction (differential error) & 0.05 \\
Resolution of beam map & 0.17 \\
Area of beam map & 0.15 \\
Total & 0.32 \\
\hline \hline
\end{tabular}

revisitation, regardless of angular orientation. Both MAXIMA I and MAXIMA II beams displayed mild beam asymmetry, which introduced systematic errors in the combination of multiple observations. Comparing the two panels in Fig. 10, the beams in MAXIMA II were noticeably more symmetric than those in MAXIMA I.

Strict correction for this asymmetry would require that the beam function be expanded in a series of spherical harmonics. ${ }^{35}$ Our approach to this problem was to compute an effective, axially symmetric beam function $B_{\ell}$ and to estimate the error incurred by such a procedure. This approach has been demonstrated to be accurate for both MAXIMA flights. ${ }^{16}$

\section{Beam characterization uncertainty}

We determined that the beam size uncertainty caused less than $4 \%$ and $11 \%$ uncertainties in the $C_{\ell}$ estimates for $\ell=410$ and 785 , respectively, and that beam asymmetry was not a dominant source of power spectrum error at any angular range. Complete beam error contributions have been reported for both MAXIMA flights. ${ }^{1,2}$

Beam map uncertainties resulted from errors in the pointing reconstruction, detector noise, the uncertainties in the bolometer electronic filters and time response, and the limited area and resolution of the beam map. We list the contributions to the total error from these sources to the measured beam FWHM for one $150 \mathrm{GHz}$ channel in Table VI. The total error is the quadrature sum of the individual contribution.

\section{DETECTORS}

The MAXIMA detector system was a 16 element bolometer array. A bolometer is a thermal detector whose electrical resistance varies as a function of temperature. This resistance is read out electrically. Thorough reviews of bolometers have been published. ${ }^{36,37}$

The audio frequency bandwidth of the bolometer signal had to be wide enough to avoid constraining the $\ell$-space coverage in the measurement of the power spectrum. The signal bandwidth was determined by the $1 / f$ knee and the high frequency rolloff in the response of the instrument. Though some of the cosmological signal appeared at frequencies as low as the telescope scan frequency of $\sim 20 \mathrm{mHz}$, it was primarily found above the $0.45 \mathrm{~Hz}$ frequency of the primary mirror modulation. Cosmological sig-

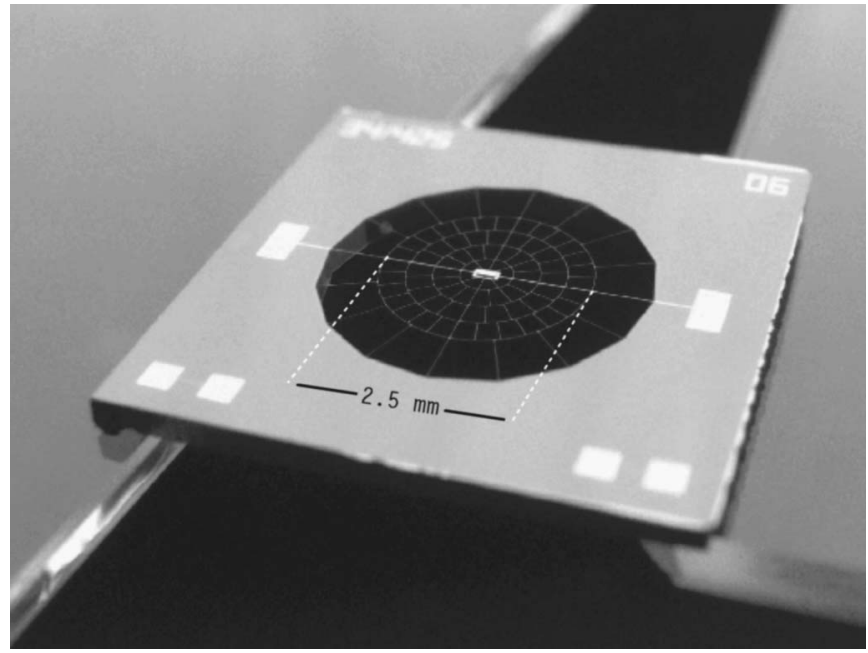

FIG. 12. The bolometer pictured is of the same design as those used in MAXIMA. The spider-web absorber had a $2.5 \mathrm{~mm}$ diameter with a $5 \%$ filling factor. The radial components within the spider-web pattern were $160 \mu \mathrm{m}$ long and $4 \mu \mathrm{m}$ wide. The NTD-Ge thermistor, a rectangle with $250 \mu \mathrm{m}$ sides, was bump bonded to the center of the web. The chip was powered through two lithographed gold leads. Mechanical support came from the electrical leads and 16 silicon nitride legs, $1 \mathrm{~mm}$ long and $5 \mu \mathrm{m}$ wide. Photograph courtesy of Bock.

nals appeared as high as the the rolloff frequency of beam window function, as determined by the telescope scan speed. We set the high frequency rolloff of the electronic filters at $20 \mathrm{~Hz}$, above the beam rolloff.

Low frequency $1 / f$ noise could have caused striping in maps. Striping was effectively suppressed by cross-linked observations; sensitivity to large scale CMB fluctuations was mostly limited by observation area (sampling variance) but was also affected by residual $1 / f$ noise.

Bolometer response time constrained the high frequency cutoff of signal band and limited the choice of scan speeds for the telescope. A slow detector response compared to the telescope scan speed would have smeared the beams on the sky, distorting the window function. It has been shown that to prevent significant distortion, bolometer response time must be at least 2.5 times faster than the time to scan a single beam size. ${ }^{38}$ This corresponded to a detector response time of $10 \mathrm{~ms}$ or less for our scans and beams.

\section{A. MAXIMA bolometers}

We used composite bolometers produced by Bock at JPL (Fig. 12). The bolometers were made with metallized mesh absorbers and neutron transmutation doped germanium (NTD-Ge) thermistors. The thermistors were obtained from J. Beeman and E. Haller at LBNL, Berkeley.

The temperature dependence of the electrical resistance of doped semiconductor thermistors has the form $R(T)$ $=R_{0} \exp (\Delta / T)^{1 / 2}$, where $\Delta \approx 10 \mathrm{~K}$ is determined by the type and level of doping in the thermistor (Ref. 39).

The characteristic temperatures for the various types of NTD-Ge allow for operation between temperatures of $20 \mathrm{mK}$ and $4.2 \mathrm{~K}$. MAXIMA bolometers were made from NTD-19, a type of NTD-Ge produced by Haller et al. at the 
TABLE VII. Average bolometer thermal properties.

\begin{tabular}{ccc}
\hline \hline $\begin{array}{c}\text { Observation } \\
\text { frequency }\langle\nu\rangle \\
(\mathrm{Hz})\end{array}$ & $\begin{array}{c}\text { Thermal } \\
\text { conductance }\langle G\rangle \\
\left(\mathrm{pW} \mathrm{K}^{-1}\right)\end{array}$ & $\begin{array}{c}\text { Temperature } \\
\text { rise }\left\langle\Delta T / T_{0}\right\rangle\end{array}$ \\
\hline 150 & 71 & 0.51 \\
240 & 290 & 0.29 \\
410 & 320 & 0.21 \\
\hline \hline
\end{tabular}

Lawrence Berkeley Laboratory which is optimized for operation at $\sim 100 \mathrm{mK}$. More information on neutron transmutation doping can be found in Ref. 40.

Electrical contacts on the NTD-Ge chips were made through ion implantation, followed by a thermal annealing process. The wafers were metallized with an $\sim 20 \mathrm{~nm}$ thick layer of $\mathrm{Cr}$ or $\mathrm{Ti}$, followed by an $\sim 150 \mathrm{~nm}$ layer of $\mathrm{Au}$ for easy attachment of wires. The wafers were then diced, etched, and passivated. Gold leads were indium bump bonded onto the metallized edges of the thermistor.

The bolometer absorbing structure was fabricated via optical lithography starting with a silicon on insulator (SOI) wafer coated with a $1 \mu \mathrm{m}$ layer of silicon nitride. The top of the silicon nitride was coated with a layer of gold whose thickness was selected to obtain an average sheet resistance of $377 \Omega /$ sq. A web with this sheet resistance absorbs $50 \%$ of the incoming radiation in one pass. ${ }^{41}$ The absorber was then patterned in a "spider-web" geometry with photoresist. A dry etch was used to remove the gold and around the pattern. The SOI was dry etched from the backside of the wafer with deep trench silicon etcher.

The spacing of the absorber legs was small enough to trap millimeter wave radiation, but was large enough to let high energy cosmic rays pass through. The absorber had a $5 \%$ filling factor, which reduced its cosmic ray cross section by $\sim 95 \%$. As previously mentioned, the optical efficiency was further increased by placing the bolometers in an integrating cavity with a characteristic depth of $\lambda / 4$.

\section{B. Noise characterization}

The major contributors to bolometer noise are photon noise, Johnson noise, thermal fluctuation noise, and noise from the first stage of amplification (amplifier noise). A thorough discussion and derivation of each noise term is found by Richards. ${ }^{37}$ Bolometer noise is generally discussed in the context of noise equivalent power (NEP) [or noise equivalent temperature (NET)] which is defined as the incident signal power (or temperature) required to generate a signal equal to the noise in a $1 \mathrm{~Hz}$ bandwidth. The individual noise sources are uncorrelated. The total noise is given by the sum of each term in quadrature.

We choose the thermal conductance $G$ required to reduce the contribution of thermal fluctuation noise compared to photon noise (Table VII). We operated the bolometers at a bath temperature of $T_{0} \approx 100 \mathrm{mK}$, which was cold enough that thermal fluctuation noise was less than the photon noise. We used low noise JFET preamplifiers. We constrained these various parameters with the help of computer-generated models, such as the one shown in Fig. 13.

We computed bolometer noise power spectra from a subset of the data during the CMB observations. We found the frequency power spectrum out to the Nyquist frequency of $104 \mathrm{~Hz}$. A typical noise power spectrum is shown in Fig. 14. The measurement can be divided into three frequency ranges. For frequencies below $\sim 100 \mathrm{mHz}$, the spectrum decreases as $1 / f$. At intermediate frequencies, the noise spectrum is white. At frequencies above $\sim 20 \mathrm{~Hz}$, the spectrum decreases because of the electronic low-pass filter and bolometer time constant.
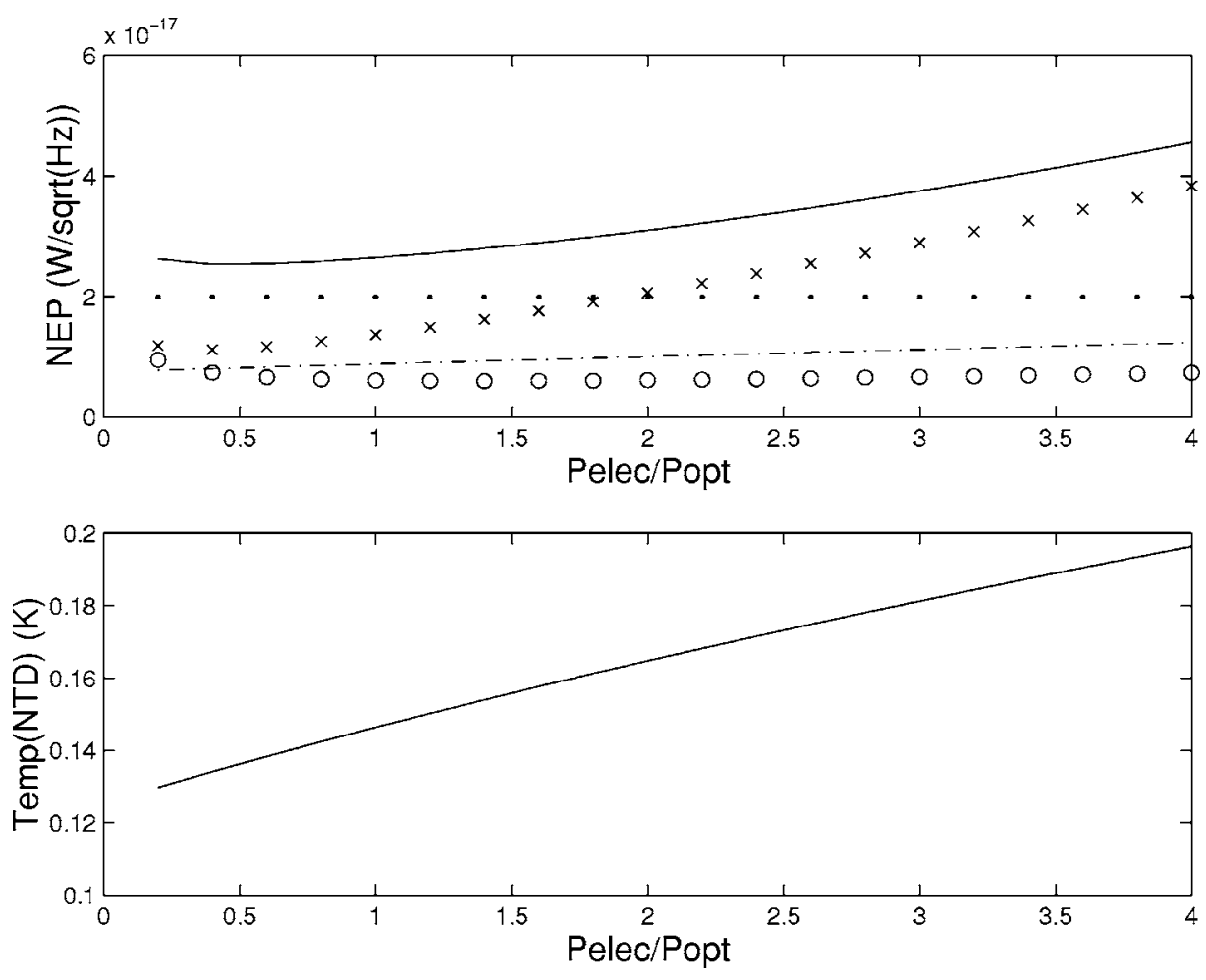

FIG. 13. Bolometer noise simulation. The top panel is a plot of the contributions to the bolometer NEP as a function of the ratio of the electrical power $P_{\text {elec }}$ to the optical power $P_{\text {opt }}$. The contributions are thermal fluctuation noise (dot-dash), Johnson noise (circles), amplifier noise (crosses), and photon noise (dotted). The solid line is the quadrature sum of the terms. The bottom panel is a plot of the thermistor temperature as a function of the same ratio of powers. We show the simulation for an operating temperature $T_{\text {base }}$ of $\sim 100 \mathrm{mK}$, an optical load $P_{\text {opt }}$ of $2 \mathrm{pW}$, an average thermal conductance $G$ of $70 \mathrm{pW} \mathrm{K}^{-1}$, and JFET voltage noise $e_{n}$ of $6 \mathrm{nV} \mathrm{Hz}^{-0.5}$. 


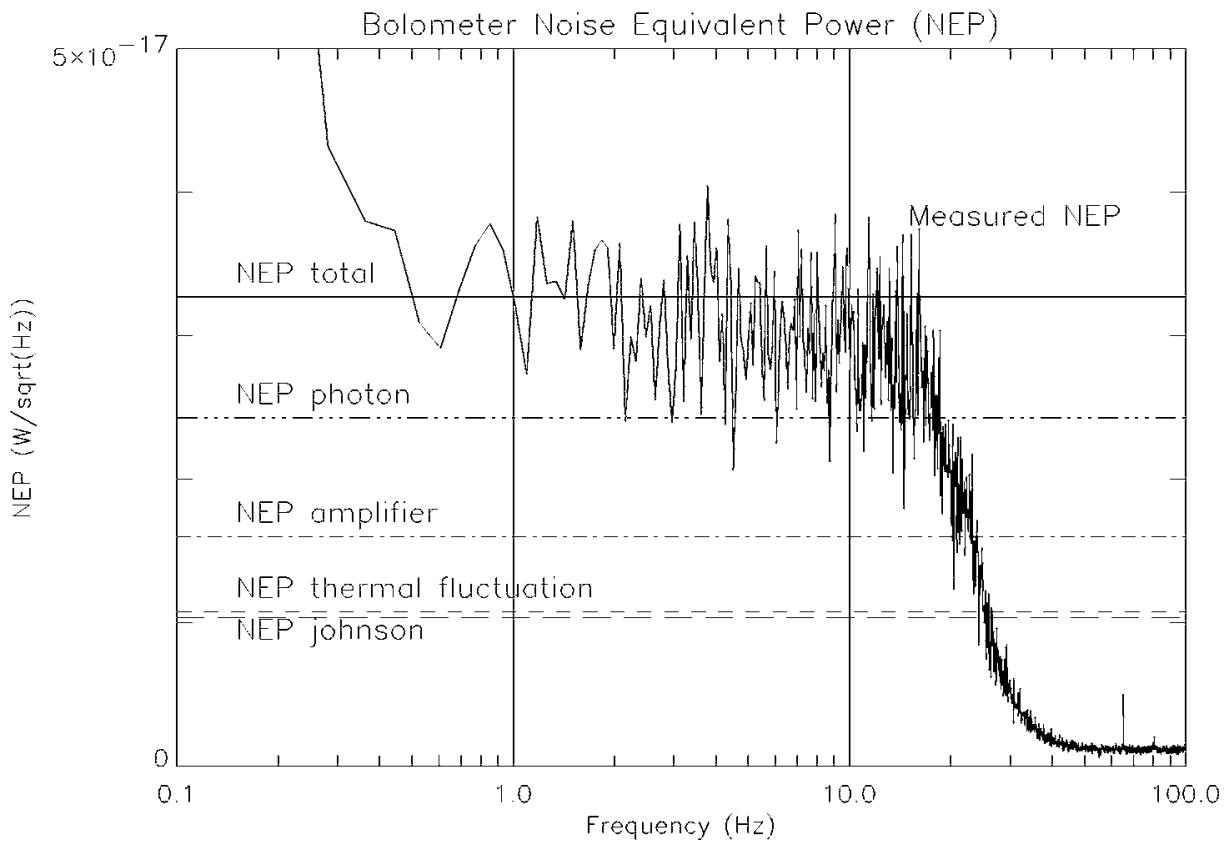

FIG. 14. The measured noise equivalent power (NEP) as a function of audio frequency for a $150 \mathrm{GHz}$ MAXIMA bolometer plotted along with theoretical NEP contributions from photon (dotted), Johnson (long dash), thermal fluctuation (short dash), and amplifier (dot-dash) noise. The total NEP (solid line) is the quadrature sum of the terms. It was believed that the low frequency noise below $0.4 \mathrm{~Hz}$ arises from temperature drifts in the cryogenic system. Electronic filters in the readout circuit cause the signal to roll off above $20 \mathrm{~Hz}$

Noise measurements for all functioning channels are presented for MAXIMA I in Table VIII and for MAXIMA II in Table IX. The voltage noise was determined from the average noise measured around a narrow audio frequency band $(7.0 \pm 0.2 \mathrm{~Hz})$ near the center of the bolometer signal band. We assigned an uncertainty of $\sim 2 \mathrm{nV} \mathrm{Hz}^{-0.5}$ to conservatively account for the nonuniformity of the white noise level over the signal band. This uncertainty did not take into account isolated peaks in the noise spectra of microphonically sensitive detectors (see discussion below). These measurements served as a diagnostic of in-flight instrumental performance. A more sophisticated noise estimation ${ }^{18}$ was used for

TABLE VIII. MAXIMA I bolometer characterization. Note that data from the channels in bold were used for Ref. 1, 2, and 5 data analysis.

\begin{tabular}{ccccrc}
\hline \hline Channel & $\begin{array}{c}\nu_{\text {obs }} \\
(\mathrm{GHz})\end{array}$ & $\begin{array}{c}V_{n} \\
\left(\mathrm{nV} \mathrm{Hz}^{-0.5}\right)\end{array}$ & $\begin{array}{c}\mathrm{NEP} \\
\left(\times 10^{-17} \mathrm{~W} \mathrm{~Hz}^{-0.5}\right)\end{array}$ & $\begin{array}{c}\mathrm{NET}_{\text {cmb }} \\
(\mu \mathrm{K} \sqrt{\mathrm{s}})\end{array}$ & $\begin{array}{c}\tau \\
(\mathrm{ms})\end{array}$ \\
\hline b14 & 150 & 16 & 2.8 & 130 & 12.5 \\
b15 & 150 & 10 & 2.0 & 80 & 12.5 \\
b24 & 150 & 18 & 4.0 & 170 & 12.5 \\
b25 & 150 & 12 & 2.4 & 80 & 10.0 \\
b34 & 150 & 12 & 1.8 & 75 & 7.0 \\
b35 & 150 & 22 & 4.6 & 200 & 9.5 \\
b44 & 150 & 38 & 6.8 & 230 & 10.5 \\
b45 & 150 & 12 & 2.2 & 80 & 9.0 \\
$150_{\text {comb }}$ & 150 & 5 & 0.9 & 35 & $\ldots$ \\
b13 & 240 & 14 & 3.4 & 380 & 10.5 \\
b23 & 240 & 14 & 6.0 & 185 & 7.0 \\
b33 & 240 & 10 & 5.4 & 140 & 9.5 \\
b43 & 240 & 24 & 9.8 & 345 & 9.0 \\
$240_{\text {comb }}$ & 240 & 7 & 2.4 & 100 & $\ldots$ \\
b12 & 410 & 18 & 6.6 & $\ldots$ & 12.0 \\
b22 & 410 & 20 & 11.0 & $\ldots$ & 8.5 \\
b32 & 410 & 24 & 7.6 & $\ldots$ & 6.0 \\
b42 & 410 & 34 & 19.0 & $\ldots$ & 5.0 \\
$410_{\text {comb }}$ & 410 & 11 & 4.4 & $\cdots$ & $\cdots$ \\
\hline \hline
\end{tabular}

finding CMB maps and angular power spectra.

The detectors were sensitive to capacitively coupled microphonic noise over the frequency range of $150-500 \mathrm{~Hz}$. This noise spectrum was measured over a frequency range of $0-500 \mathrm{~Hz}$ while exciting the cryostat with a broad-band mechanical vibration generator. Measurements revealed both monoenergetic and broad resonances $(10-30 \mathrm{~Hz}$ wide). The overall noise amplitude for each channel varied considerably over the detector array. Wiring between the bolometers and the JFET amplifiers were stiffened and shortened, and the entire detector assembly was further tethered to the cooled optics supports inside the cryostat with tensioned Kevlar

TABLE IX. MAXIMA II bolometer characterization. Note that data from the channels in bold were used for Ref. 4 and 42 data analysis.

\begin{tabular}{ccccrc}
\hline \hline Channel & $\begin{array}{c}\nu_{\text {obs }} \\
(\mathrm{GHz})\end{array}$ & $\begin{array}{c}V_{n} \\
\left(\mathrm{nV} \mathrm{Hz}^{-0.5}\right)\end{array}$ & $\begin{array}{c}\mathrm{NEP} \\
\left(\times 10^{-17} \mathrm{~W} \mathrm{~Hz}^{-0.5}\right)\end{array}$ & $\begin{array}{c}\mathrm{NET}_{\mathrm{cmb}} \\
(\mu \mathrm{K} \sqrt{\mathrm{s}})\end{array}$ & $\begin{array}{c}\tau \\
(\mathrm{ms})\end{array}$ \\
\hline b14 & 150 & 28 & 7.6 & 345 & 12.5 \\
b15 & 150 & 10 & 2.4 & 65 & 11.5 \\
b24 & 150 & 10 & 3.0 & 100 & 10.5 \\
b25 & 150 & 10 & 2.8 & 80 & 12.0 \\
b34 & 150 & 10 & 2.6 & 100 & 6.5 \\
b35 & 150 & 8 & 2.4 & 90 & 8.0 \\
b44 & 150 & 28 & 8.8 & 350 & 8.5 \\
b45 & 150 & 10 & 2.8 & 85 & 8.5 \\
$150_{\text {comb }}$ & 150 & 4 & 1.0 & 35 & $\ldots$ \\
b13 & 240 & $\ldots$ & $\ldots$ & $\ldots$ & $\ldots$ \\
b23 & 240 & 10 & 8.0 & 165 & 5.0 \\
b33 & 240 & 12 & 6.0 & 170 & 8.5 \\
b43 & 240 & $\ldots$ & $\ldots$ & $\ldots$ & $\cdots$ \\
$240_{\text {comb }}$ & 240 & 88 & 4.8 & 120 & $\ldots$ \\
b12 & 410 & 20 & 18 & $\ldots$ & 14.0 \\
b22 & 410 & 12 & 16 & $\ldots$ & 6.5 \\
b32 & 410 & 12 & 14 & $\ldots$ & 2.5 \\
b42 & 410 & 20 & 24 & $\ldots$ & 3.0 \\
$410_{\text {comb }}$ & 410 & 7 & 8.1 & $\cdots$ & $\cdots$ \\
\hline \hline
\end{tabular}


synthetic fiber string [Dupont (U.S.A.)]. These mechanical changes reduced microphonic sensitivity in most channels. The ac voltage bias strategy described in Sec. V F enabled flexibility in selecting a $40 \mathrm{~Hz}$ signal band (between 250 and $450 \mathrm{~Hz}$ ) in which microphonic pickup was not apparent in the demodulated noise spectrum for most detectors. A subset of the detector channels displayed significant microphonic noise both in laboratory tests and during cosmological observations (three channels for MAXIMA I and four channels for MAXIMA II).

As the overall NEP for each channel was largely determined by a subset of the best performing bolometers, only these few were used for the published MAXIMA data analysis. Maps of CMB temperature anisotropy were made using three $150 \mathrm{GHz}$ channels and one $240 \mathrm{GHz}$ channel for MAXIMA I and from four $150 \mathrm{GHz}$ channels for MAXIMA II. Data from these channels and from one $410 \mathrm{GHz}$ channel were used for systematic tests, notably spectral discrimination of foreground signals.

For most MAXIMA I channels, noise during CMB observations was stationary at the $10 \%-20 \%$ level. For the channels with high microphonic sensitivity, NET values were calculated using a section of the data without microphonic noise. These channels were not included in the final analysis. Most time streams exhibited some degree of noise correlated with the spatial modulation of the primary mirror. This mirror synchronous signal proved to be stationary over long enough time scales, and we developed a formal template subtraction in subsequent analysis. ${ }^{18}$ Low frequency noise rose as $1 / f$, with knees between 0.2 and $0.6 \mathrm{~Hz}$. Detector noise was Gaussian except near the chopper frequency. Frequencies below $0.1 \mathrm{~Hz}$ were poorly sampled and have been marginalized over in data analysis.

For MAXIMA II, low frequencies noise rose more steeply than $1 / f$ in all channels, with knees ranging from 1 to $3 \mathrm{~Hz}$. The source of the noise was unknown, and the time scale over which it was stationary varied between channels. In addition, mirror synchronous noise appeared in the higher observation frequency channels, especially during the beginning of the second $\mathrm{CMB}$ observation. The reported NEPs and NETs were not a complete reflection of the overall noise performance. Analysis of MAXIMA II data ${ }^{12,42}$ required marginalization over frequencies below $0.2 \mathrm{~Hz}$.

\section{Response time characterization}

Measurements of the bolometer response times and noise were made during both the MAXIMA I and MAXIMA II flights. We determined the response times by deconvolving a single pole low-pass filter, whose characteristic frequency was a free parameter, from bolometer time streams while scanning the primary telescope back and forth in azimuth across a planet. In analysis, the bolometer response time parameter was adjusted until the left and right going scans spatially coincided. This measurement had a statistical error of $0.50 \mathrm{~ms}$. The measurements are presented in Tables 8 and 9 for MAXIMA I and MAXIMA II, respectively. We replaced some of the detectors between the two flights, which explains the difference in response time for many channels. In
TABLE X. MAXIMA cryogenic systems. Note that numbers are quoted for flight conditions.

\begin{tabular}{ccccc}
\hline \hline Cooler & Liquid $\mathrm{N}_{2}$ & ${ }^{4} \mathrm{He}$ & ${ }^{3} \mathrm{He}$ & ADR \\
\hline Temperature (K) & 50 & $2-3$ & 0.35 & 0.1 \\
Hold time (h) & 24 & $>30$ & $>36$ & 12 \\
Thermal cycle & Open & Open & Closed & Closed \\
\hline \hline
\end{tabular}

all cases, the measured response time was small enough to prevent significant beam smearing from the telescope modulation.

\section{RECEIVER AND ELECTRONICS}

The MAXIMA cryostat housed the secondary optics, the bolometer array and preamplifiers, an optical calibration source, and the cryogenic system. There were also a number of diagnostic devices including "dark" detector channels not exposed to the CMB and a variety of internal temperature monitors.

A cross sectional drawing of the receiver is shown in Fig. 6. The bottom section of the receiver contained the baffled, liquid ${ }^{4} \mathrm{He}$ cooled optics and the internal relative calibration source. The middle section held the bolometers and bolometer feedhorns, the cryogenic JFET preamplifiers, and the thermal switches. The top section of the receiver contained the cryogenic systems, with the low temperature refrigerators surrounded by the liquid ${ }^{4} \mathrm{He}$ tank.

The cryostat was manufactured by Infrared Laboratories (U.S.A.) (model HDL14). It had an outer diameter of $40.5 \mathrm{~cm}$ and a length of $112.5 \mathrm{~cm}$. Liquid $\mathrm{N}_{2}$ and ${ }^{4} \mathrm{He}$ were held in stainless steel walled tanks, which were structurally supported by G-10 laminate standoffs. The cold plate for each tank was made of $1.9 \mathrm{~cm}$ thick gold-plated oxygen-free high-conductivity (OFHC) copper, which provided excellent thermal contact. The joint between the copper cold plate and the stainless steel wall of the ${ }^{4} \mathrm{He}$ tank degraded after repeated thermal cycling over six years. We believed that the weakness of the joint was due to differential thermal contraction over the large size of the tank. A replacement tank and cold plate were both constructed from aluminum to minimize differential thermal contraction.

\section{A. Cryogenics}

The detector array was cooled to $\sim 100 \mathrm{mK}$ during flight via a four-stage refrigeration process. The array was housed inside an evacuated cryostat and was cooled by an adiabatic demagnetization refrigerator (ADR) backed by a closedcycle ${ }^{3} \mathrm{He}$ sorption refrigerator, a pumped open-cycle liquid ${ }^{4} \mathrm{He}$ bath, and a pumped open-cycle liquid $\mathrm{N}_{2}$ bath. These cooling systems are summarized in Table $\mathrm{X}$.

A 131 liquid $\mathrm{N}_{2}$ tank cooled an outer layer of radiation shielding to $77 \mathrm{~K}$. During the flights, this temperature dropped to $\sim 50 \mathrm{~K}$ when the liquid $\mathrm{N}_{2}$ tank was exposed to vacuum. The liquid $\mathrm{N}_{2}$ temperature radiation shields were covered with thin, low emissivity aluminum foil.

Inside the liquid- $\mathrm{N}_{2}$-cooled space was a 211 liquid ${ }^{4} \mathrm{He}$ tank and an additional layer of shielding at liquid ${ }^{4} \mathrm{He}$ temperature. The outer shell of the cold optics box served as part 
of this radiation shielding. The outside of these shields was low emissivity aluminum, while the inner surfaces were coated with the blackening mixture described in Sec. III A. The blackened interior absorbed high temperature radiation that leaked past the shields.

Within the liquid ${ }^{4} \mathrm{He}$ temperature space were the optics, the detectors, the JFET preamplifiers, the sub-Kelvin coolers, and a variety of thermometers. The optics and most electrical components were thermally linked to the cold plate.

Various locations in the liquid- ${ }^{4} \mathrm{He}$-cooled stage ranged in temperature from 4 to $6 \mathrm{~K}$, depending on thermal load and proximity to the helium tank. When the liquid ${ }^{4} \mathrm{He}$ was exposed to vacuum, for testing and in flight, these temperatures dropped to $2-3 \mathrm{~K}$. This caused a significant drop in the background radiation loading the bolometers.

Inset into the liquid ${ }^{4} \mathrm{He}$ tank were the $\mathrm{ADR}$ and the liquid ${ }^{3} \mathrm{He}$ refrigerator. All wiring entering the receiver was heat sunk to the liquid ${ }^{4} \mathrm{He}$ and liquid $\mathrm{N}_{2}$ tanks and was made of low thermal conductivity stainless steel leads.

\section{1. ${ }^{3} \mathrm{He}$ refrigerator}

We designed and constructed a large capacity ${ }^{3} \mathrm{He}$ sorption refrigerator for the MAXIMA cryostat. The closed-cycle refrigerator consisted of 42 STP liters of ${ }^{3} \mathrm{He}$ of $99.995 \%$ purity, an activated charcoal sorption pump, a copper condenser, and a copper evaporator which was hermetically coupled to the condenser with a thin walled stainless steel bellows. The bellows was structurally reinforced with three thin walled Vespel polyimide SP1 tubes (Dupont [U.S.A.)] connecting the evaporator to the ${ }^{4} \mathrm{He}$ cold plate.

Due to size constraints within the cryostat, the sealed ${ }^{3} \mathrm{He}$ refrigerator was coupled to an external tank to prevent overpressurization. The ${ }^{3} \mathrm{He}$ pressure in the combined vessels at room temperature was $745 \mathrm{kPa}$, a factor of 4 smaller than the elastic limit of the bellows. The condenser was directly coupled to the ${ }^{4} \mathrm{He}$ cold plate. The sorption pump consisted of $131 \mathrm{~g}$ of activated charcoal. The grains of charcoal were glued with Stycast 2850 FT epoxy [Emerson \& Cuming Microwave Products (U.S.A.)] to closely spaced copper fins which increased the pump area. Calculations showed that optimal sorption was achieved with at least $3 \mathrm{~g}$ of charcoal per STP liter of gas.

The pump was thermally linked to the ${ }^{4} \mathrm{He}$ cold plate with an ultrahigh purity tin wire. The thermal conductivity of tin was known to decrease rapidly with temperature. By design, the tin wire was a poor thermal link for temperatures above $10 \mathrm{~K}$. The refrigerator was cycled by first heating the charcoal to $40 \mathrm{~K}$ for $\sim 30 \mathrm{~min}$, which desorbed the ${ }^{3} \mathrm{He}$ from the pump. After heating, the charcoal and tin slowly cooled back to the ${ }^{4} \mathrm{He}$ cold plate temperature. Meanwhile, the desorbed atoms condensed into liquid and pooled into the evaporator. Once the charcoal had cooled below $10 \mathrm{~K}$, it pumped on the ${ }^{3} \mathrm{He}$, which cooled the liquid to $\sim 300 \mathrm{mK}$. The refrigerator maintained this base temperature for $36-48 \mathrm{~h}$ with an unpumped ${ }^{4} \mathrm{He}$ bath and had a cooling capacity of $25 \mathrm{~J}$.

\section{2. $A D R$}

We used an ADR developed in Berkeley as a prototype for the Spitzer Space Telescope (SST). This ADR was flown multiple times on the MAX experiment. The details of the construction and testing of the MAX/MAXIMA ADR are published elsewhere. $^{43,44}$

The ADR consisted of a paramagnetic salt pill inside a superconducting magnet. The salt pill was made from $40 \mathrm{~g}$ of ferric ammonium alum (FAA) and was supported within the magnet coil with a Kevlar synthetic fiber [Dupont (U.S.A.)] string suspension that was thermally intercepted by the ${ }^{3} \mathrm{He}$ refrigerator cold stage. The ${ }^{4} \mathrm{He}$ cooled superconducting $\mathrm{Nb}-\mathrm{Ti}$ coil generated a peak field of $2.5 \mathrm{~T}$ with a current of $6.2 \mathrm{~A}$. The ADR was thermally cycled with a remotely commandable current controller. During cycling, the heat of magnetization was transferred to the ${ }^{3} \mathrm{He}$ refrigerator cold stage. The ADR had a laboratory tested hold time of $17 \mathrm{~h}$ and a flight-tested hold time of $12 \mathrm{~h}$ at $\sim 100 \mathrm{mK}$ with an $\sim 90 \%$ duty cycle. The ADR had a cooling capacity of $0.093 \mathrm{~J}$.

The wiring from the outside of the cryostat to the magnet was designed to control thermal conductivity and electrical power dissipation. The magnet current entered the cryostat through hermetic connectors and was carried by bundles of standard 22 AWG copper wire heat sunk to the liquid $\mathrm{N}_{2}$ cold plate. Each bundle of wire was soldered to a high- $T_{c}$ superconducting lead [yttrium barrium copper oxide (YBCO)] [Eurus Monoco (U.S.A.)] clamped between the liquid $\mathrm{N}_{2}$ cold plate and the top of the liquid ${ }^{4} \mathrm{He}$ tank. The magnet current was then carried by Formvar coated copper clad $\mathrm{Nb}-\mathrm{Ti}$ superconducting wire which was heat sunk to the side of the liquid ${ }^{4} \mathrm{He}$ tank. The $\mathrm{Nb}-\mathrm{Ti}$ leads were potted in Eccosorb CR-124 epoxy [Emerson \& Cuming Microwave Products (U.S.A.)] before entering the ${ }^{4} \mathrm{He}$ cold-plate area, in order to filter RFI.

All of the wiring from the high- $T_{c}$ leads to the magnet coil was superconducting during cryogenic operation. Any significant electrical power dissipation was dumped into the liquid $\mathrm{N}_{2}$.

\section{B. Bolometer wiring}

The signals for each bolometer entered the cryostat via hermetic connectors. Once inside the cryostat, the signals were carried by a harness of low thermal conducting stainless steel wire [Cooner Wire (U.S.A.)] heat sunk at its midpoint to the liquid $\mathrm{N}_{2}$ thermal stage. The signals were then carried in wire striplines of $50 \mu \mathrm{m}$ gold-plated Ni wire, fabricated by Tayco Co. (U.S.A.), which were potted in Eccosorb CR-124 [Emerson \& Cuming (U.S.A.)]. These modules were heat sunk to the ${ }^{4} \mathrm{He}$ cold plate and blocked radio frequency interference signals (described in Sec. V E).

The wiring between the RFI filters and the JFET modules (Sec. V F) was conventional Teflon-coated multistrand copper. The signals were then delivered from the JFET module to the $\sim 100 \mathrm{mK}$ array via Formvar coated $50 \mu \mathrm{m}$ platinum tungsten wire [California Fine Wire (U.S.A.)], which had a low thermal conductance. We bundled and twisted the wires for the bolometers around four thin walled G-10 laminate tubes that were rigidly connected to both the JFET mod- 
ule and the array. The tubes were thermally intercepted by an OFHC copper heat strap from the $0.3 \mathrm{~K}$ thermal stage.

The wiring continued from the G-10 tubes to each photometer on four G-10 laminate printed circuit boards with $0.25 \mathrm{~mm}$ wide copper traces. We covered both sides of the circuit board with electrically insulated aluminum tape to reduce the emissivity of the array. More details on wiring inside the MAXIMA cryostat are found by Winant. ${ }^{14}$

\section{Housekeeping thermometry}

We monitored the internal temperatures with various cryogenic thermometers; we used silicon diodes at $77 \mathrm{~K}$, carbon composition thermistors at 2-4 $\mathrm{K}$, a $\mathrm{RuO}_{2}$ thermometer for the ${ }^{3} \mathrm{He}$ refrigerator $(0.3 \mathrm{~K})$, and a germanium thermistor for the ADR $(0.1 \mathrm{~K})$. Thermometer leads entered the cryostat through the same hermetic connectors as the bolometer signals. They were then carried by the same stainless steel wire harness and Eccosorb RFI filter module into the cold-plate area. Each device was wired to the RFI module with low thermal conductivity Teflon coated $0.125 \mathrm{~mm}$ manganin wire [California Fine Wire (U.S.A.)]. The wires for the $0.1 \mathrm{~K}$ thermometer were heat sunk midway on the ${ }^{3} \mathrm{He}$ refrigerator cold stage.

\section{Internal relative calibrator}

Bolometer responsivity varied over the duration of a flight, primarily because of variations in the temperature of the $\sim 100 \mathrm{mK}$ ADR. These variations were monitored using a stable internal calibration source (i.e., a stimulator) consisting of a thin nickel-chromium layer $\left(2 \times 2 \mathrm{~mm}^{2}\right)$ deposited on a sapphire substrate which was connected to the ${ }^{4} \mathrm{He}$ stage by a weak thermal link. The impedance of the film-substrate combination was chosen to match the impedance of free space so as to radiate efficiently when heated. The stimulator was mounted inside the cold optics box and was fitted with a light pipe to illuminate the focal plane array from just outside the optical path. The illumination of the array was not uniform, with detectors closer to the calibrator receiving about twice the flux of the more distant detectors. A heating current was applied for $10 \mathrm{~s}$, every $20 \mathrm{~min}$ during flight. During heating, the film warmed to $\sim 50 \mathrm{~K}$ with a time constant of $\sim 1 \mathrm{~s}$. The stability of the current source and the measured variations in the temperature of the ${ }^{4} \mathrm{He}$ bath ensured that variations in the power radiated by the stimulator were $<1 \%$.

The absolute flux was not well measured, so the stimulator was used purely for monitoring of responsivity variations over time. Absolute calibration was obtained from celestial sources (the CMB dipole and planets). The use of stimulator data in detector calibration is discussed in Sec. VI C.

\section{E. RFI protection}

During flight, several radio transmitters were used for telemetry. Each radiated $15-40 \mathrm{~W}$ at frequencies of 1.5-3.0 GHz. Extensive filtering was required to prevent interference in the detectors from these sources.

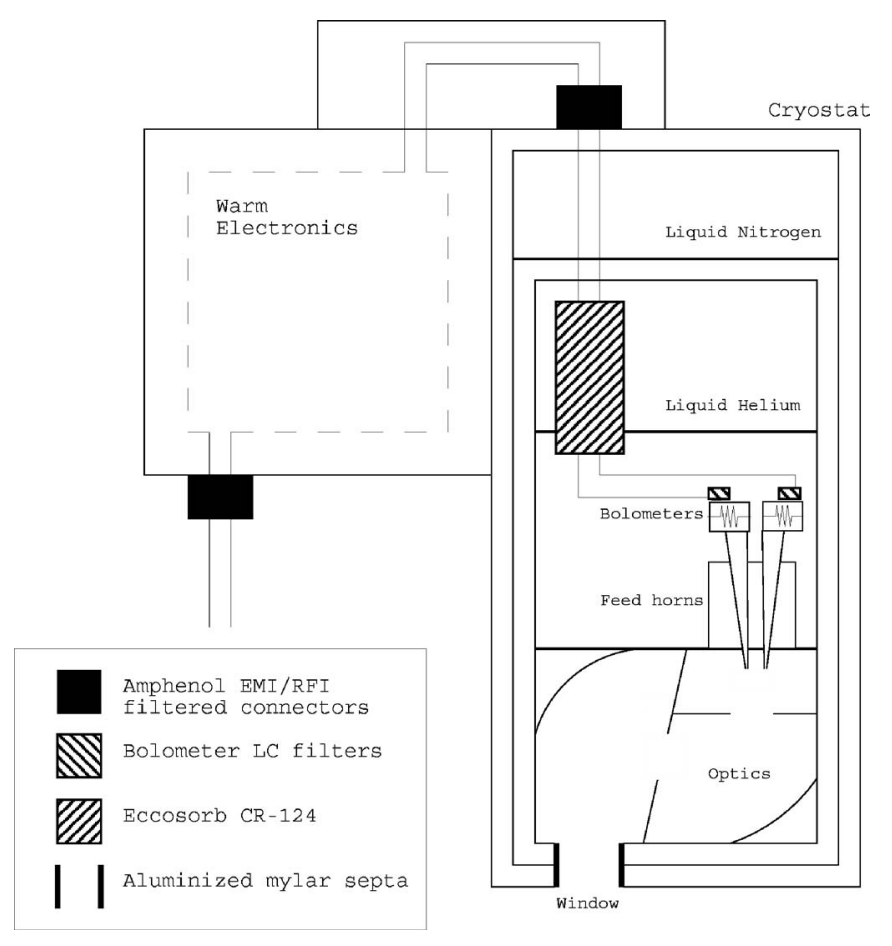

FIG. 15. The bolometers (shown with resistor symbols) were shielded from electrical RFI by three different types of filters in series, shown as filled rectangles. RFI was prevented from transmitting coaxially between the temperature baffles by contiguous layers of aluminized Mylar placed around the optical path at the cryostat window. The cryostat and the warm electronics shield acted as Faraday cages.

The receiver itself consisted of three metallic shells which served as partial Faraday cages. External cabling was also fully enclosed in a metallic shell. All cables between the receiver and the readout electronics and all cables exiting the readout electronics passed through commercial rf filtered connectors [Amphenol (U.S.A.) FPT02 series, $60 \mathrm{~dB}$ attenuation at $1.0 \mathrm{GHz}$. Within the receiver, all wiring was potted in $27 \mathrm{~cm}$ of Eccosorb CR-124 [Emerson \& Cuming Microwave Products (U.S.A.)] which acted as a radio frequency low-pass filter $(30 \mathrm{~dB}$ attenuation at $1.0 \mathrm{GHz})$.

The optical window acted as a high-pass filter for ambient RFI, which had a measured cutoff of $\sim 2.5 \mathrm{GHz}$. Higher frequency RFI in the optical path was blocked by taping a cylindrical sleeve made from multiple layers of $25 \mu \mathrm{m}$ sheets of aluminized Mylar around the perimeter of the optical path near the cryostat window with aluminum tape (Fig. 15). This attenuated the coaxial transmission of $\mathrm{rf}$ between the shields without significantly compromising the thermal isolation between the different temperature stages inside the cryostat.

Each individual bolometer was enclosed in a RFI-tight cavity. The optical opening into the cavity was small enough to prevent RFI at frequencies below $100 \mathrm{GHz}$ from radiatively coupling onto the bolometers. The bolometer signals were filtered at the cavity wall by $L C$ feed through filters [muRata Industries (Japan)]. Calculations of the high frequency performance of these filters including distributed reactances were made, and were consistent with the improvements reported below.

The aluminized mylar septa and bolometer cavity $L C$ 
filters were incorporated after MAXIMA I and before MAXIMA II. Tests revealed that both optical and dark (test) detectors with $L C$ filters showed dramatic improvement at $1<f<2 \mathrm{GHz}$. The septa and $L C$ circuits contributed at least $20 \mathrm{~dB}$ suppression of pickup in this frequency range. The $L C$ circuits were shown not to introduce measurable suppression or phase shifts in the signal band of the detectors.

This evidence supported the model that during MAXIMA I, RFI leaked in through the window, around the temperature baffles, and then coupled into the bolometer wiring. This was believed to have caused a small, scan synchronous signal seen by some MAXIMA I detectors.

\section{F. Bias and readout electronics}

The analog bolometer readout electronics were designed by Hristov from CalTech. The bolometers were ac biased to reduce low frequency noise contributions from the electronics (e.g., $1 / f$ noise in the JFET amplifiers). The ac bias did not cause fluctuations in the bolometer temperature, because $f_{\text {bias }} \gg 1 / 2 \pi \tau_{\text {bolo. }}$. All detectors were biased at the same frequency by a single sine wave oscillator which could be tuned from 250 to $400 \mathrm{~Hz}$, well above the $0-20 \mathrm{~Hz}$ audio band. We set this frequency before each flight to minimize microphonic noise.

The bias voltage was independently set for each detector to between 50 and $100 \mathrm{mV}$. Bias current variations were reduced by two $40 \mathrm{M} \Omega$ load resistors in series with each bolometer. The largest fractional change in bias current, observed during the MAXIMA I planet scan, was of order $10^{-5}$.

We reduced the microphonic sensitivity of the receiver by coupling the bolometers to low output impedance cryogenic amplifiers. The amplifiers for each detector were a matched pair of cooled monolithic-dual silicon EJ-TIA JFETs [Infrared Laboratories (U.S.A.)] in a differential emitter follower circuit. The JFET pairs were packaged in standard transistor cans with internal thermal isolation and were cooled by a weak thermal link to the liquid ${ }^{4} \mathrm{He}$ bath, for an operating temperature of $150 \mathrm{~K}$. Each JFET pair consumed $300 \mu \mathrm{W}$ of power and contributed $6-10 \mathrm{nV} \mathrm{Hz}^{-0.5}$ to the total noise per channel.

The bolometer signals were further processed by readout electronics outside the cryostat. They were preamplified with an AD624 operational amplifier, bandpass filtered around the bias frequency, and rectified with an AD630 lock-in amplifier referenced to the bias signal.

Bolometer resistance fluctuations appeared in the sidebands of the bias frequency, as determined by the telescope scan speed. Given the primary mirror modulation frequency of $0.45 \mathrm{~Hz}$ and peak velocity of $4 \mathrm{deg} \mathrm{s}^{-1}$ (or $\sim 25$ beam FWHM per second), the data were in the range of $0.1-20 \mathrm{~Hz}$ around the bias frequency. Signals outside the band were suppressed by four pole Butterworth filters with a characteristic frequency of $19.96 \pm 0.61 \mathrm{~Hz}$ (averaged over all 20 readout channels).

Finally, the rectified signal was amplified with a gain of 1800 and split to two outputs. We removed the offset from one signal stream using a single pole high-pass filter with a characteristic frequency of $14.9 \pm 0.7 \mathrm{mHz}$ (averaged over all 20 readout channels). This signal was further amplified by a
TABLE XI. Calibration linearity. Note that quoted values were derived from the maximum of the signal for MAXIMA I (before the slash) and MAXIMA II (after the slash). Ranges represent variations between detectors. For the dipole calibration the upper limit on nonlinearity was $\sim 0.05 \%$.

\begin{tabular}{lcc}
\hline \hline & $\begin{array}{c}\text { Planet } \\
\text { responsivity } \\
\text { change } \\
\text { (MAXIMA I/MAXIMA II) }\end{array}$ & $\begin{array}{c}\text { Stimulator } \\
\text { responsivity } \\
\text { change }\end{array}$ \\
\hline $150 \mathrm{GHz}$ & $0.7 \%-2.5 \% / 0.2 \%-0.5 \%$ & $0.1 \%-0.5 \% / 0.1 \%-1.5 \%$ \\
$240 \mathrm{GHz}$ & $1.0 \%-6.1 \% / 0.2 \%-0.4 \%$ & $0.2 \%-4.0 \% / 0.1 \%-0.5 \%$ \\
$410 \mathrm{GHz}$ & $1.9 \%-7.7 \% / 0.4 \%-1.0 \%$ & $0.5 \%-3.3 \% / 0.3 \%-2.7 \%$ \\
\hline \hline
\end{tabular}

factor of 36, yielding a net gain of 65000. Overall, the gain of the readout circuit had a temperature stability of $<10^{-5}\left({ }^{\circ} \mathrm{C}\right)^{-1}$.

\section{G. Supporting electronics and telemetry}

High gain bolometer signals were digitally sampled with 16 bit resolution every $4.8 \mathrm{~ms}$. Other data including cryostat housekeeping, ambient temperatures, bolometer bias monitors, primary mirror position, electronics status, and low gain bolometer signals were sampled every $19.2 \mathrm{~ms}$. The data were multiplexed and transmitted to a ground based data recording station over a $1.8 \mathrm{GHz}$ radio downlink at a data rate of $160 \mathrm{kbits} \mathrm{s}^{-1}$. A separate $1.5 \mathrm{GHz}$ downlink transmitted for the data from the pointing system and from balloon facility support systems. Video signals from pointing sensors were transmitted at 2-3 GHz, but were not transmitted during $\mathrm{CMB}$ scans to reduce the ambient radio frequency radiation. Control of the telescope from the ground was maintained via intermittent command signals at $\sim 500 \mathrm{MHz}$.

The digitizing and multiplexing data acquisition system was built at the Lawrence Berkeley National Laboratory, while telemetry and commanding systems were provided by the National Scientific Balloon Facility.

\section{CALIBRATION}

Absolute responsivity calibration used two known sources during each MAXIMA flight: the CMB dipole and a planet. Measurements of the CMB dipole gave the best absolute calibration for the 150 and $240 \mathrm{GHz}$ detectors. Observations of planets (Jupiter in MAXIMA I and Mars in MAXIMA II) were used to calibrate the $410 \mathrm{GHz}$ detectors and were used as a consistency check for the dipole calibration. The internal millimeter wave source (the stimulator described in Sec. V D) was used to periodically measure responsivity changes. The effects of the optical loads of the calibration sources on detector responsivity are summarized in Table XI.

The MAXIMA I data had a calibration error of $4 \%$, while the MAXIMA II data had a calibration error of $3 \%$. These were the most accurate calibrations achieved by any suborbital CMB experiment at the time of publication. The MAXIMA calibrations were consistent with that of WMAP. $^{12}$ 


\section{A. CMB dipole}

The dipole was the main calibrator for the 150 and $240 \mathrm{GHz}$ detectors, using the amplitude measurement of the COBE satellite. ${ }^{45}$ Dipole observations were carried out by rotating the telescope in azimuth with an azimuthal angular velocity of $20 \mathrm{deg} \mathrm{s}^{-1}$. The observed signal from the dipole was a sine wave at $56 \mathrm{mHz}$. We observed the dipole at elevations of $50^{\circ}$ in MAXIMA I (to avoid sidelobe response from the Moon at lower elevation) and at $32^{\circ}$ in MAXIMA II. During MAXIMA I the dipole, including the motion of the Earth around the Sun, had a magnitude of $3.195 \mathrm{mK}$ and was tilted $20^{\circ}$ from the horizon; the projected amplitude over the scan region was $2.04 \mathrm{mK}$. During MAXIMA II the dipole had a magnitude of $3.010 \mathrm{mK}$ and was tilted $48^{\circ}$ from the horizon; the projected amplitude over the scan region was $1.15 \mathrm{mK}$.

The rotating scan pattern of the dipole observation was sensitive to parasitic signals from galactic dust. The dust signal was modeled by extrapolating published maps ${ }^{23,46}$ to our frequencies. The dust signal was much smaller than the dipole signal at $150 \mathrm{GHz}$, except near the galactic plane. Data within $5^{\circ}$ of the galactic plane were neglected in data analysis. Elsewhere, the dust model was fitted to the data along with the dipole model. Overall normalization was taken as a free parameter to account for uncertainties in the frequency extrapolation of the dust signal. In practice, the dust model did not affect the dipole calibration due to its low amplitude and lack of a dipolelike spatial component.

We began the dipole observation near the beginning of the flight, while the telescope was still ascending from $\sim 21.5 \mathrm{~km}$ to the final observing altitude of $\sim 38.5 \mathrm{~km}$. An additional signal was observed during the first third of the observation (altitude $<30 \mathrm{~km}$ ). We believe that this signal was atmospheric for four reasons: (1) it was highly correlated in all the optical bolometers; (2) it was spectrally consistent with atmospheric emission, being larger for the higher frequency detectors; (3) it was spatially stable on the scale of a few minutes, but varied on longer scales; (4) the magnitude of the signal declined steadily with altitude.

The atmospheric signal was corrected using data from the $410 \mathrm{GHz}$ bolometers. These data, which were relatively insensitive to the dipole and sensitive to the atmospheric signal, were used as a template for the signal in the 150 and $240 \mathrm{GHz}$ data. A correction was applied for the CMB sensitivity of the $410 \mathrm{GHz}$ detectors, as calibrated by planet observations. As with galactic dust, we found that fitting the atmospheric signal did not affect our final dipole calibration values. It did, however, increase calibration uncertainty, because of noise in the $410 \mathrm{GHz}$ data used to model the effect.

\section{Dipole data analysis}

During each flight, the CMB dipole was observed for $\sim 30$ min (100 rotations). For each detector, the effects of electronic filters and bolometer time constants were first deconvolved from the entire data stream. Data from each rotation were then fitted independently according to the model
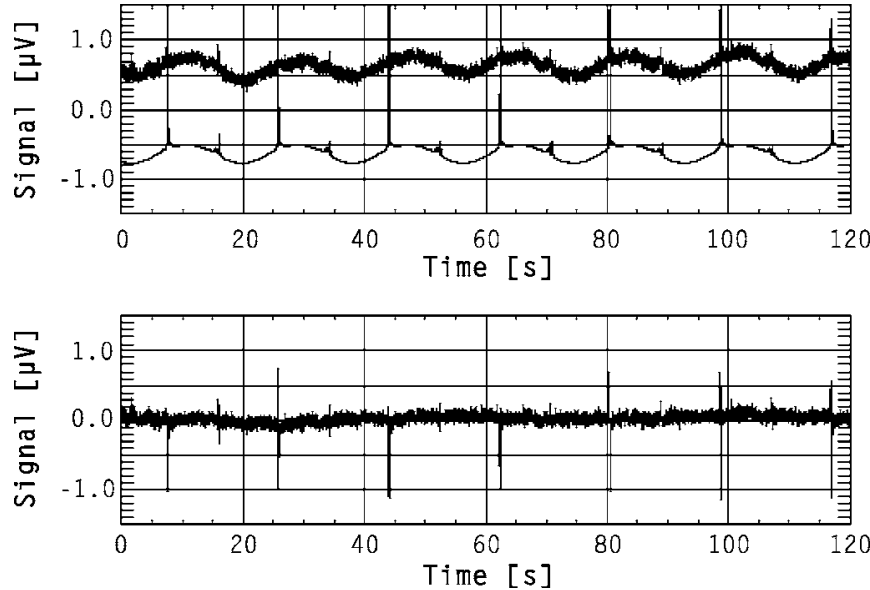

FIG. 16. MAXIMA II dipole data and fit. Top panel: The top trace is the data from a $150 \mathrm{GHz}$ bolometer during observations of the CMB dipole. An overall gradient is removed and the offset is arbitrary. The sinusoidal signal is the CMB dipole modulated by the rotation of the telescope $(\sim 18 \mathrm{~s}$ period). The large spikes were caused by emissions from dust near the galactic plane. The lower trace is a fitted model curve, including the CMB dipole and a galactic dust map. Bottom panel: The difference between the model and the fit in the top panel is shown. The pointing accuracy was not good enough to model the data from the galactic plane accurately. These regions were not included in the fit.

$$
T_{\text {detector }}=\left(A T_{\mathrm{CMB}, \text { model }}\right)+\left(B T_{\text {dust }}\right)+\left(C N_{\text {drift }}\right)+D,
$$

in which $T_{\text {detector }}$ is the time stream of detector data in voltage units, $T_{\mathrm{CMB} \text {,model }}$ is the $\mathrm{CMB}$ dipole model in units of temperature contrast, $T_{\text {dust }}$ is the galactic dust model, $N_{\text {drift }}$ is linear drift, and $A, B, C$, and $D$ are fitting constants. $A$ is the calibration of the detector to CMB signals.

For MAXIMA I the atmospheric signal was taken into account by modifying the fit to

$$
\begin{aligned}
T_{\text {detector }}= & \left(A T_{\mathrm{CMB}, \text { model }}\right)+\left(B T_{\text {dust }}\right)+\left(C N_{\text {drift }}\right)+D \\
& +\left(E T_{410}\right),
\end{aligned}
$$

where $T_{410}$ is the time stream data from a $410 \mathrm{GHz}$ detector and $E$ is an additional fitting parameter. Because the $410 \mathrm{GHz}$ data did have some very small sensitivity to the $\mathrm{CMB}, A$ was no longer an unbiased calibration. To account for this, the $410 \mathrm{GHz}$ data were first calibrated using planet data and the parameter $A$ was corrected,

$$
A^{\prime}=A-\left(E \operatorname{cal}_{410}\right),
$$

where $\mathrm{cal}_{410}$ is the planet-based calibration of the $410 \mathrm{GHz}$ data and $A^{\prime}$ is the true calibration of the low frequency channel. In practice the $410 \mathrm{GHz}$ term did not affect calibration values by more than $0.5 \sigma$, though it did increase uncertainties. The correction from $A$ to $A^{\prime}$ had a negligible effect on both the calibration and the calibration uncertainty due to the small value of the $E$ parameter.

Each rotation yielded a calibration value ( $A$ or $A^{\prime}$ above) and an associated error range. These were combined statistically, with $2 \sigma$ outliers excluded. Data from $\sim 80$ rotations were analyzed from each flight, with 2-8 excluded as outliers for each detector. Data from the MAXIMA II dipole observation are shown in Fig. 16. 


\section{Sources of dipole calibration error}

Dipole calibration uncertainty $(1 \%-4 \%$ depending on the detector) was dominated by detector noise at the dipole observation frequency of $56 \mathrm{mHz}$. The fitting routine was found numerically to reject noise beyond a fractional bandwidth of $\sim 0.5$. Noise was effectively reduced by a further factor of $\sqrt{2}$ by the known phase of the dipole model. Raw detector noise near $56 \mathrm{mHz}$ for a $150 \mathrm{GHz}$ bolometer was typically $150 \mathrm{nV} \mathrm{Hz}^{-0.5}$. Considering bandwidth and phase constraints, this yielded an expected $\sim 20 \mathrm{nV}$ noise level for a single dipole fit. For a typical $150 \mathrm{GHz}$ detector the amplitude of the dipole response was $\sim 70 \mathrm{nV}$. We therefore expected a statistical uncertainty of $\sim 30 \%$ from a single rotation.

Detector noise was the only source of statistical uncertainty in the calibration and could be estimated directly from the scatter of the individual, single rotation calibrations. Such analysis yielded single rotation statistical uncertainties of $10 \%-30 \%$ for $150 \mathrm{GHz}$ detectors in both flights. These numbers were somewhat lower than predicted due to imperfect understanding of detector noise at very low frequencies. An integration of 80-90 dipole observations per flight provided total statistical uncertainties of $1.4 \%-4.2 \%$ for $150 \mathrm{GHz}$ detectors in MAXIMA I and $1.1 \%-2.5 \%$ in MAXIMA II. Because the CMB responsivity of the $240 \mathrm{GHz}$ detectors was $60 \%-70 \%$ that of the $150 \mathrm{GHz}$ detectors, they had a proportionally higher statistical error. When combining data from multiple detectors, we used the highest statistical uncertainty of the combined channels. For MAXIMA I, the modeling of the atmospheric signal increased the final calibration uncertainty by only about $0.5 \%$.

In addition to statistical uncertainty, there were a number of known systematic effects, though none had a significant impact on the calibration. First, dipole pointing reconstruction was accurate to $\sim 10^{\prime}$ and contributed only $\sim 0.1 \%$ to the calibration uncertainty. Second, the signal from the dipole was small enough that bolometer saturation was negligible. Third, the dipole model derived from the COBE measurement was accurate to $0.68 \%$. Finally, an $\sim 15 \mathrm{mHz}$ single-pole high-pass filter in the bolometer readout electronics was characterized to $\sim 0.3 \%$ at the dipole scan frequency. The temperature dependence of this filter was tested in the laboratory and could have caused at most a $1 \%$ shift in the filter pole frequency in flight. This maximum shift would have caused only a $0.08 \%$ change in response magnitude and $0.16^{\circ}$ change in phase.

\section{B. Planets}

In each flight observations were made of a planet: Jupiter in MAXIMA I and Mars in MAXIMA II. The planet observation procedure has been described in Sec. III C 1. Responsivity calibration was obtained from the maximum voltage response. Expected signals were derived from published measurements and models of planet temperatures and emissivities, ${ }^{47-49}$ combined with the spectral response of the detectors. A correction was applied for beam dilution, the fraction of the telescope beam filled by the planet. Jupiter had an angular diameter of 46.5" during MAXIMA I and Mars had an angular diameter of 12.7" during MAXIMA II.
The dilution factors varied from $3.4 \times 10^{-3}$ to $4.4 \times 10^{-3}$ for MAXIMA I and from $3.1 \times 10^{-4}$ to $4.1 \times 10^{-4}$ for MAXIMA II. This correction was the dominant error source for the planet calibration in both flights.

Additional corrections of roughly 5\% for MAXIMA I and $1 \%$ for MAXIMA II were applied for the reduction in responsivity caused by the optical load from the planet (Table XI). This effect was neglected in the initial MAXIMA I data analysis and caused an apparent small systematic discrepancy between the dipole and Jupiter calibrations. This discrepancy was within the errors of the Jupiter calibration and did not affect the CMB map or power spectrum.

\section{Planet calibration error sources}

Because the 150 and $240 \mathrm{GHz}$ detectors were calibrated from the CMB dipole, errors in the planet calibrations did not affect the CMB power spectrum. We calculated these calibration uncertainties to interpret systematic and foreground tests using the $410 \mathrm{GHz}$ detectors and as a consistency check on the dipole calibration of the lower frequency detectors. Dipole and planet calibrations were statistically consistent within the uncertainties of each.

The dominant error term for the planet calibration was the uncertainty in the beam dilution factor. The uncertainty in the integrated beam response was 5\%-10\%. In addition, there was a possibility of small, broad sidelobes that were not measured in the beam maps. We assigned an uncertainty of $10 \%$ from beam shape errors. Beam shape error, especially that due to broad sidelobes, was partially correlated between detectors because of their shared optics.

Uncertainties in the effective brightness temperature of the planets contributed 5\% to the calibration error. The brightness temperature of Mars was modeled to this accuracy, both by extrapolation from high frequency observations ${ }^{48}$ and by physical modeling. ${ }^{49}$ The atmospheric properties for Jupiter have made modeling relatively difficult. Our expected Jupiter signal was based on published brightness ratios between Jupiter and Mars. ${ }^{47}$ The planet temperature uncertainty was fully correlated between all the detectors.

Measurements of the detector spectra contributed $1 \%-2 \%$ error at $150 \mathrm{GHz}, 3 \%-7 \%$ at $240 \mathrm{GHz}$, and $2 \%-3 \%$ at $410 \mathrm{GHz}$. Measurements of the peak planet voltage contributed 1\%-4\% error; one detector in MAXIMA II was anomalously noisy, increasing this term to $\sim 10 \%$. Uncertainty in the bolometer saturation was negligible.

\section{Time dependent calibration}

The responsivity of bolometers varied by $1 \%-2 \%$ for a change of $1 \mathrm{mK}$ in the thermal reservoir temperature. The temperatures varied by $\sim 6 \mathrm{mK}$ over the course of data collection in MAXIMA I and by $\sim 21 \mathrm{mK}$ in MAXIMA II. Responsivity variations were monitored using the internal millimeter wave source (stimulator) described in Sec. VD. Temperature variations over the period of a stimulator excitation were negligible. To obtain relative calibration values from stimulator events, we began by subtracting an overall gradient from each event to remove the effects of detector drift. We then performed a linear fit between pairs of stimu- 


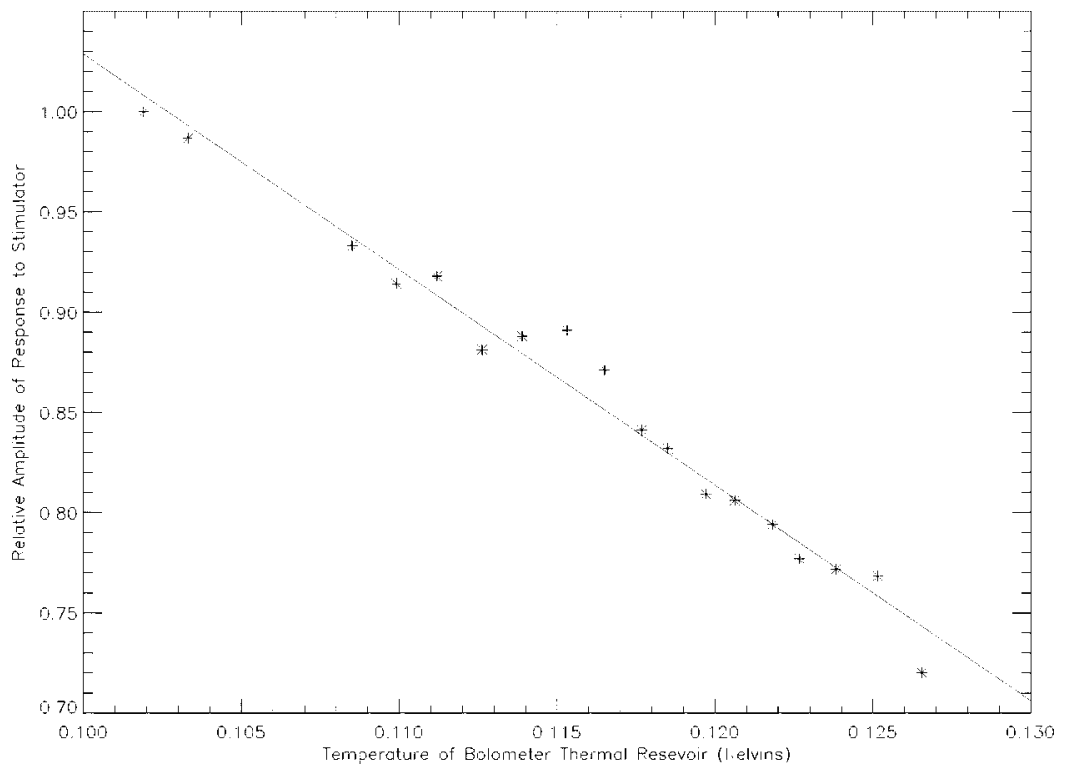

FIG. 17. Temperature dependence of the responsivity of a $150 \mathrm{GHz}$ detector. These data were collected during MAXIMA II. The point near $0.127 \mathrm{~K}$, measured shortly after sunrise, showed less responsivity than would be expected from the nighttime data because of the higher radiation loading. During MAXIMA I, the temperature of the thermal reservoir only varied from 98 to $104 \mathrm{mK}$. lator events. The slope of this fit was the calibration ratio between the events, while the offset of the fit was simply an offset in the detector data. Once the relative calibration at each stimulator event was known, we fit the values to a linear function of the temperature of the bolometer thermal reservoir (Fig. 17). This fit was combined with the absolute calibration to obtain the overall calibration as a function of time throughout the flight (Sec. VI D).

\section{Relative calibration error analysis}

The relative calibration between stimulator events was affected by random variations (detector noise or stimulator instability), but was not affected by systematics that were consistent between stimulator events, such as uncertainties in the spectra of the detectors and the beam filling of the stimulator signal. We treated the relative calibration as a linear function of temperature and took the optical load during the flight as constant. Assumptions were supported by bolometer models ${ }^{14,50}$ and contributed negligibly to calibration error. The reduction in bolometer responsivity due to the large optical load of the stimulator was nearly stable (Table XI). Though it did vary with bolometer temperature, this variation contributed less than $0.1 \%$ error to the relative calibration. Random errors in the comparison of stimulator events were $1 \%-2 \%$. Instabilities in the stimulator current accounted for $<0.5 \%$ of this, while detector noise accounts for the rest.

\section{Combined calibration}

The overall calibration for each detector was obtained by combining an absolute calibrator with the relative calibration. The absolute calibrator the CMB dipole for 150 and $240 \mathrm{GHz}$ detectors and was the planet scan for $410 \mathrm{GHz}$ detectors. The relative calibration was based on the temperature of the bolometer thermal reservoir and the responsivitytemperature relation obtained in Sec. VI C. Temperature was monitored continuously.

The overall calibration error was the combined error from the absolute calibrator and the relative calibration.
Relative calibration error varied over the course of the flight. Quoted values are based on averages over the CMB observations. Relative calibration error was subdominant for MAXIMA II (1\%-2\%) and was negligible for MAXIMA I $(<0.1 \%)$. The published MAXIMA I data were conservatively assigned the highest calibration uncertainty of the detectors used, which was $4 \%$.

\section{SCANS AND POINTING}

\section{A. Scan strategy}

Each CMB observation was conducted at a fixed elevation, while the telescope beams were moved in azimuth. The azimuthal modulation defined one dimension of the roughly rectangular scan region. The rotation of the sky over the duration of the observation defined the other dimension.

The azimuthal motion consisted of two independent modulations. The primary mirror rotation provided a relatively fast modulation with a frequency of $0.45 \mathrm{~Hz}$ and a peak-peak span of $4.0^{\circ}$. The motion of the entire telescope provided slower modulation at frequencies from 12 to $25 \mathrm{mHz}$, depending on the scan parameters, with peakpeak spans of $4.5^{\circ}-9.0^{\circ}$. The fast modulation prevented our data from being significantly corrupted by low frequency noise. Taken together, the two made our data relatively robust against modulation synchronous parasitic signals.

The two CMB scans of each flight observed the same region of the sky, but at different times and at different elevation angles. The rotation of the sky between these observations caused the two scans to be tilted relative to each other in sky-stationary coordinates. The average angles of cross-linking were $22^{\circ}$ for MAXIMA I and $27^{\circ}$ for MAXIMA II. The two azimuthal modulations, the rotation of the sky, and the cross-linked revisitation were uncorrelated and were on radically different time scales, minimizing the effects of potential scan-correlated systematics.

Depth of integration was set by the total width of the combined azimuthal modulation and the rotation rate of the 
TABLE XII. Pointing performance.

\begin{tabular}{|c|c|c|c|c|}
\hline $\begin{array}{c}\text { CMB } \\
\text { observation }\end{array}$ & $\begin{array}{l}\text { MAXIMA I } \\
\text { scan } 1\end{array}$ & $\begin{array}{l}\text { MAXIMA I } \\
\text { scan } 2\end{array}$ & $\begin{array}{l}\text { MAXIMA II } \\
\text { scan } 1\end{array}$ & $\begin{array}{c}\text { MAXIMA II } \\
\text { scan } 2\end{array}$ \\
\hline Maximum scan speed $\left(\operatorname{deg~s}^{-1}\right)$ & 0.29 & 0.30 & 0.29 & 0.26 \\
\hline rms velocity error $(\prime \prime)$ & 0.022 & 0.028 & 0.043 & 0.057 \\
\hline
\end{tabular}

sky. The average integration times per beam size were $\sim 2.5 \mathrm{~s}$ in MAXIMA I and $\sim 2.2 \mathrm{~s}$ in MAXIMA II. This led to average expected noise levels of $\sim 60 \mu \mathrm{K}$ per beam size area for our best single detector and $\sim 40 \mu \mathrm{K}$ for our published combination of four MAXIMA I detectors. In practice, integration was several times longer in the center of the observed region and shorter near the edges.

\section{B. The attitude control system}

The pointing system, or attitude control system (ACS), served both to control the orientation of the telescope in flight and to acquire the data needed for postflight pointing reconstruction. The pointing system consisted of attitude sensors, a central feedback loop control computer, and motors. Some of the easily interpreted sensors were used in pointing control, while the most precise sensors, the CCD cameras, were used after the flight for pointing reconstruction. The ACS was originally constructed at IROE-CNR (now IFAC-CNR) and Universitá di Roma La Sapienza, in Italy. The system was similar to that used in the BOOMERanG experiment. ${ }^{51}$

A computer read data from the various sensors, applied a digital feedback algorithm, and set the power level for the motors. Each of these tasks was performed once every $96 \mathrm{~ms}$, synchronously with the bolometer data acquisition system. Signals from most sensors were sampled each cycle. Data from the CCD cameras were processed by a separate computer, and were passed to the control computer every two cycles (192 ms). Global Positioning System (GPS) data (absolute time and position) were updated once per second.

Pointing normally followed one of several preprogramed flight schedules. Remote (ground-based) commanding was used to switch between, modify, or override schedules. In addition, remote commanding was used to modify control loop gains, to make adjustments to sensor calibrations, and to set parameters for the CCD image processing.

Feedback control was based on azimuthal rotation velocity, as measured by a rate gyroscope which had an accuracy of $\sim 0.01 \mathrm{deg} \mathrm{s}^{-1}$. Though they were very sensitive, the gyroscopes had substantial low frequency drifts, primarily due to ambient temperature changes. Drifts were calibrated once per gondola scan period and had little impact on pointing control. Two other gyroscopes, which measured pitch and roll velocities, were not used in feedback control. The performance of the feedback control loop is summarized in Table XII.

Absolute azimuth was measured in real time using a two axis magnetometer. The magnetometer was extremely precise $\left(<0.5^{\prime}\right)$ in differential measurement, but was highly nonlinear due to the magnetic properties of the gondola. Preflight measurements were used to calibrate the magnetometer to an accuracy of $\sim 30^{\prime}$.

Absolute elevation was measured in real time by an optical angle encoder between the inner assembly (receiver and primary mirror) and the outer frame of the telescope. The accuracy of this measurement depended on the balancing of the telescope $\left(\sim 0.1^{\circ}\right)$ and on long time scale pendulum motion $\left(\sim 0.5^{\circ}\right.$, which varied over tens of minutes). The differential accuracy of this elevation measurement was $\sim 1^{\prime}$.

\section{1. $C C D$ cameras}

The CCD star cameras provided the most accurate measurement of telescope orientation and were used for postflight reconstruction. One camera was mounted on the inner telescope assembly and was boresighted with the telescope beams when the primary mirror was in its central position. The second camera was positioned on the outer frame approximately $40^{\circ}$ to the right of the boresight, at a fixed elevation angle of about $31^{\circ}$, so that north celestial pole star (Polaris) was in the camera's field of view during CMB observations. The boresighted camera data were used for the final pointing reconstruction and were accurate to $\sim 0.5^{\prime}$. The secondary camera was only accurate to $\sim 15^{\prime}$, due to the offset of the measurement from the telescope boresight combined with pendulum motion of the telescope. Secondary camera data were used to identify stars in the primary camera. The boresighted camera had a field of view of $7.17^{\circ}$ $\times 5.50^{\circ}$ with a pixel size of $0.84^{\prime} \times 0.69^{\prime}$. The resolution was further improved to $\sim 0.5^{\prime}$ in flight by software interpolation. The secondary camera had a larger field of view $\left(14.34^{\circ}\right.$ $\left.\times 11.00^{\circ}\right)$ and lower resolution $\left(\sim 1.0^{\prime}\right)$.

The boresighted camera reliably detected stars of apparent visual magnitude $m_{v} 5.0$ or brighter. Stars of visual magnitude 5.0-6.0 were detected intermittently, and stars dimmer than visual magnitude 6.0 were rarely detected. This sensitivity was sufficient to detect stars in all of the MAXIMA I scan region and $\sim 80 \%$ of the MAXIMA II scan region. Source brightness was not an issue for the secondary camera, which always viewed Polaris (visual magnitude $=2.0$ ).

Image processing introduced a delay of $\sim 200 \mathrm{~ms}$ to the camera data. The cameras internally sampled the CCD chips at $30 \mathrm{~Hz}$, asynchronous to the rest of the system. This caused a jitter up to $33 \mathrm{~ms}$ in the image processing delay, for an overall delay of $200 \pm 16 \mathrm{~ms}$ between pointing data and bolometer data. At MAXIMA gondola scan speeds, $200 \mathrm{~ms}$ translated to between $2^{\prime}$ and $5^{\prime}$, which was a significant fraction of the $10^{\prime}$ beam size and was taken into account in the 


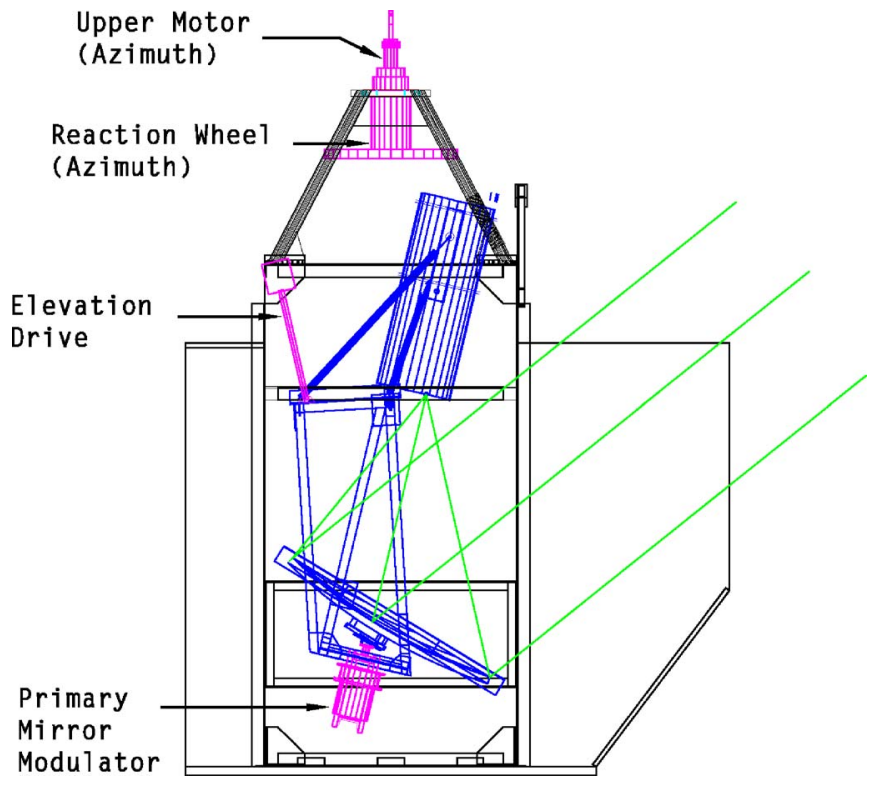

FIG. 18. (Color online) Two motors near the top of the telescope controlled the azimuthal orientation by driving against a reaction wheel and the cables from the balloon, respectively. A linear actuator and servoarm tilted the inner assembly, pointing the telescope in elevation. A motor below the primary mirror modulated it at relatively high speed $\left(0.45 \mathrm{~Hz}, \pm 2^{\circ}\right.$ amplitude $)$ in azimuth. The frames shown on the front and back of the telescope were baffled to reduce far sidelobe response.

pointing reconstruction. The $\pm 16 \mathrm{~ms}$ jitter translated to $\sim 0.15^{\prime}$ rms pointing uncertainty.

\section{Motors}

Three motors were used to point the MAXIMA telescope and a fourth was used to modulate the primary mirror. They are shown schematically in Fig. 18. Two motors, located near the top of the telescope frame, were used for pointing in azimuth. One of these drove a reaction wheel with a moment of inertia of $10 \mathrm{~kg} \mathrm{~m}^{2}(\sim 0.5 \%$ that of the telescope). The other torqued against suspension cables connected to the balloon, which had a much greater moment of inertia than the telescope. Both motors were direct drive (ungeared), had a peak torque of $33.9 \mathrm{Nm}$, and received a maximum power of $\sim 50 \mathrm{~W}$ from the control system. The light reaction wheel provided fast response, while the other motor kept the speed of the reaction wheel low by transferring angular momentum to the balloon. The rotational velocities of these motors were monitored by tachometers.

Elevation control was provided by a geared motor connected to a linear actuator arm. The arm was fixed between the outer assembly of the telescope frame and the inner assembly of the receiver and primary mirror. The inner assembly was balanced about the rotation axis, so the static load on this motor was very small.

Motor power was determined in a digital control loop. Pointing control in azimuth and elevation was not strongly coupled and may be considered separately. In azimuth, we used a rotational velocity-based proportional feedback system based on measurements from a rate gyroscope. In CMB scans, the target velocity was constant, except during turnarounds in the scan direction. During turnarounds the target velocity varied linearly with time. The absolute position was not used directly in the control loop; it was instead used to trigger these turnarounds. An additional feedback term proportional to the rotational velocity of the flywheel was applied to the upper motor, slowly transferring flywheel angular momentum to the balloon. The elevation control formula was based on the measured angle of the telescope inner assembly relative to the outer frame. In this case, the power to the elevation drive was determined by an angular positionbased proportion-derivative feedback loop.

The primary mirror was continuously rotated from side to side about the axis indicated in Fig. 6 in Sec. III. The motion was a rounded triangle scan with an amplitude of $\pm 2^{\circ}$ and a frequency of $0.45 \mathrm{~Hz}$. This modulation superimposed on that of the entire telescope yielded the scan pattern in Fig. 19. The resulting scan pattern in RA and Dec is shown in Figs. 20 and 21. The mirror was actuated by a de motor with solid state PID control electronics. The motor was obtained commercially and the control electronics were built at Berkeley. Mirror position was controlled to an accuracy of $1^{\prime}$.

Because the mirror rotation axis was not vertical, there was also a small modulation in elevation-a bowed pattern in which the extremes of the mirror modulation rose slightly in elevation. The elevation motion could in principle have led to a scan synchronous signal in the bolometers due to atmospheric emission. In practice, the elevation motion $\left(<2^{\prime}\right)$ was not large enough to generate a detectable signal.

\section{Pointing reconstruction}

The complete pointing reconstruction for one detector for both MAXIMA flights can be seen in Fig. 5. Pointing reconstruction was based primarily on data from the CCD cameras. Often, pointing could be determined simultaneously from two different stars in the same field of view. In these cases the discrepancy between the two pointing solutions was used to estimate the overall error in the CCD measurement. The typical discrepancy was $\sim 0.5^{\prime}$ (Table XIII). Between camera measurements, the telescope typically moved $\sim 8^{\prime}$. In the azimuth, data from the rate gyroscope were integrated to find intervening positions. As a test of accuracy, the azimuthal data were reinterpolated numerically and were compared to the gyroscope-based interpolation. The difference between the two was used to estimate the pointing error introduced by interpolation. Though the rms discrepancy between these two methods was very small, the distribution had extreme outliers corresponding to regions of the sky with few bright stars. Interpolated regions with a difference of greater than 3.3' (one-third of the FWHM beam size) were not used in data analysis.

In the elevation direction, the rate gyroscopes were more difficult to calibrate. However, the motion of the telescope in elevation was extremely slow and small, so these data were safely interpolated numerically.

Finally, the effect of the primary mirror modulation was included. The angle of the primary mirror was measured to several arc sec by a linear variable differential transformer (LVDT). The LVDT data were calibrated both before and during flight using data from the planet observation. The motion of the primary mirror moved the telescope beams primarily in azimuth. However, there was a small motion in 


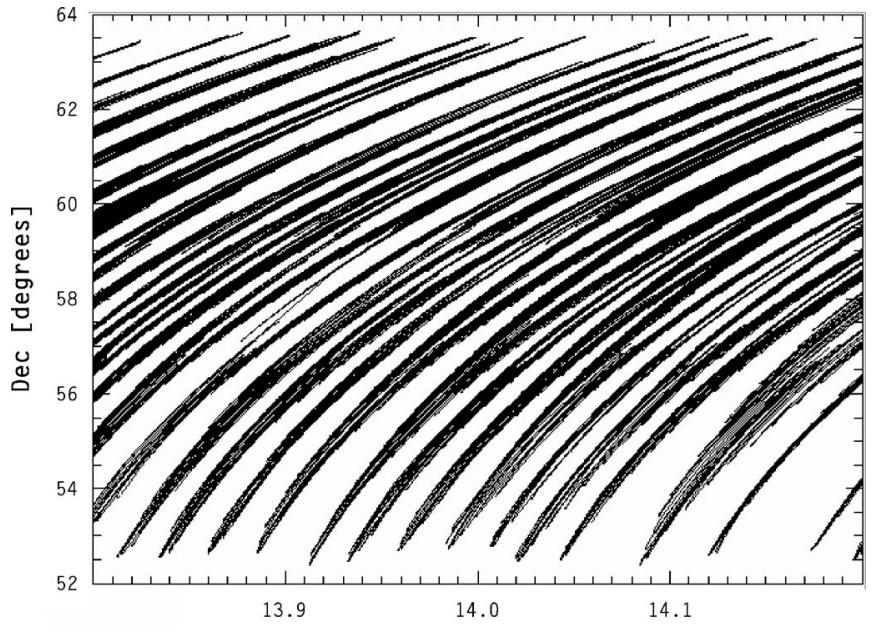

RA [hours]

FIG. 20. A MAXIMA II scan pattern plotted in RA and declination, combining the azimuthal modulations with the rotation of the sky. Lines of constant elevation span the plot in an arc from the lower left to the upper right and move with the rotation of the sky. The gaps seen in this scan pattern are less than half the telescope beam width.

elevation which was much more difficult to calibrate and was a source of pointing uncertainty. The elevation motion depended upon the zero position of the mirror. This was measured to $\sim 1^{\circ}$, which led to a conservative pointing uncertainty of about $0.8^{\prime} \mathrm{rms}$.

The $0.8^{\prime}$ uncertainty of the primary mirror modulation was the largest source of error in the MAXIMA pointing solution. Though purely systematic, the scan pattern and cross-linking tended to blur out the effect. In addition, other sources of pointing uncertainty further randomized the total error. The overall pointing error was approximated as a $1^{\prime}$ Gaussian blur. End to end simulations have demonstrated that such a blur leads to a $2.5 \%$ reduction in the CMB power spectrum at $\ell=500$ and that this reduction scaled roughly as

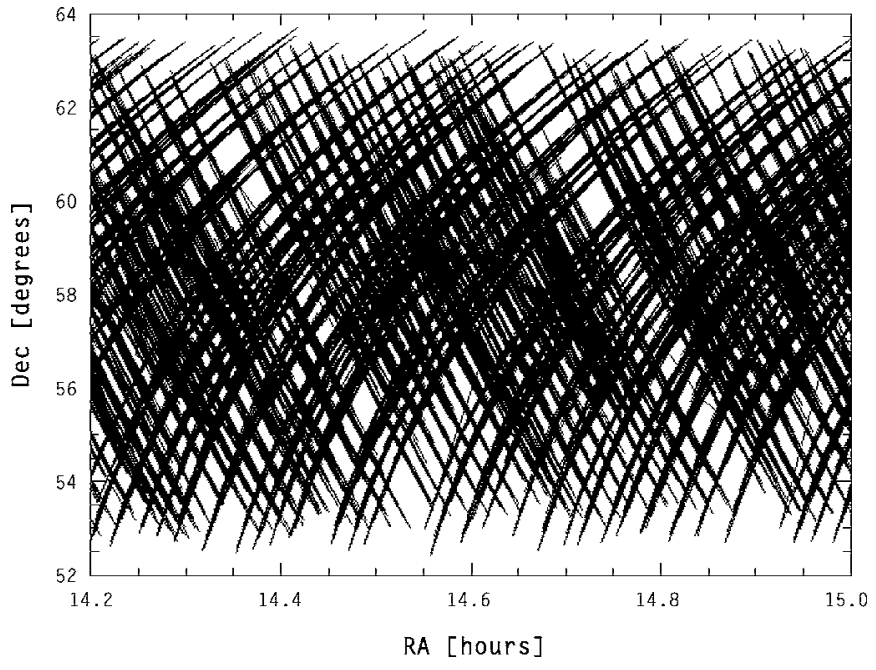

FIG. 21. The cross-linked scan pattern from MAXIMA II, consisting of two scans similar to that shown in Fig. 20. The average cross-linking angle was $27^{\circ}$.

$\ell^{2}$, over the range where the fractional reduction was much less than 1 .

Pointing uncertainty was a subdominant source of CMB power spectrum error at all values of $\ell$. While it was possible to compensate the power spectrum for the reduction caused by pointing error, we have not done so because it was relatively small, and because our model of the pointing error as Gaussian was not exact.

\section{SUMMARY AND DISCUSSION}

The performance of MAXIMA I provided an important measurement of $\mathrm{CMB}$ temperature anisotropy on subdegree scales. High resolution maps, power spectra over multipoles of $35 \leqslant \ell \leqslant 1235$, and cosmological parameter estimates were generated from data from 5 of the 16 photometers. $^{1-5}$

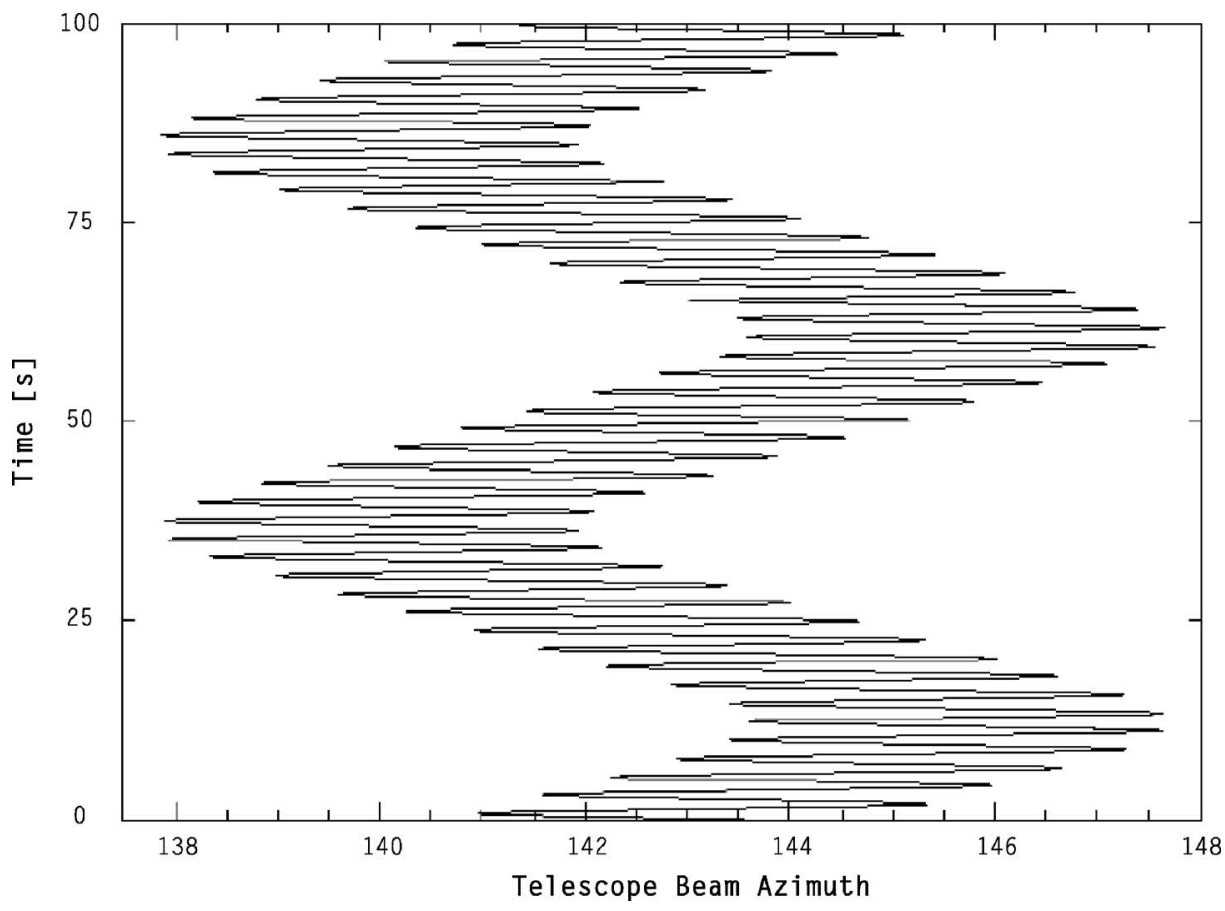

FIG. 19. A simulation of the double modulation in azimuth. The $x$ axis is the azimuthal position of the telescope beams, while the $y$ axis is time. The slower modulation was caused by the motion of the entire telescope, while the faster modulation was caused by the rotation of the primary mirror about the optic axis. 
TABLE XIII. Pointing reconstruction uncertainties.

\begin{tabular}{|c|c|c|c|c|c|}
\hline & \multicolumn{3}{|c|}{ Random errors } & \multicolumn{2}{|c|}{ Systematic errors } \\
\hline & $\begin{array}{c}\text { CCD } \\
\text { camera }^{\text {a }}\end{array}$ & $\begin{array}{l}\text { Camera } \\
\text { timing }^{\mathrm{b}}\end{array}$ & Interpolation $^{\mathrm{c}}$ & $\begin{array}{l}\text { Detector } \\
\text { offset }^{\mathrm{d}}\end{array}$ & $\begin{array}{c}\text { Primary } \\
\text { modulation }\end{array}$ \\
\hline MAXIMA I & $0.46^{\prime}$ & $0.15^{\prime}$ & $<0.001^{\prime}$ & $0.25^{\prime}$ & $0.81^{\prime}$ \\
\hline MAXIMA II & $0.58^{\prime}$ & $0.15^{\prime}$ & $<0.001^{\prime}\left(\sim 1^{\prime}\right)$ & $0.25^{\prime}$ & $0.81^{\prime}$ \\
\hline \multicolumn{6}{|c|}{$\begin{array}{l}\text { a CCD camera-error in image analysis. } \\
{ }^{b} \text { Camera timing - uncertainty in CCD sampling time relative to detector sampling, multiplied by telescope scan } \\
\text { speed. } \\
\text { 'Interpolation- }<0.001 \text { ' interpolation error applies to all of MAXIMA I and } \sim 80 \% \text { of MAXIMA II. The rest } \\
\text { of MAXIMA II had very few stars, increasing this term. } \\
\text { d Detector offset-uncertainty in the offset between the telescope beams and the camera boresight. } \\
\text { e Primary modulation—uncertainty in mirror orientation. }\end{array}$} \\
\hline
\end{tabular}

Both intermediate and final results passed a series of systematic tests, ${ }^{12,42}$ probing contamination from instrumental noise, from foregrounds, or from the data analysis pipeline.

The $225 \mathrm{deg}^{2}$ area of the sky that was scanned during the MAXIMA II flight in 1999 overlapped with $50 \mathrm{deg}^{2}$ of the area scanned during MAXIMA I and was larger by about a factor of 2, see Fig. 5. The expected detector performance and scan strategy were similar between the two flights. The scientific results from MAXIMA II, including power spectra and cross correlations with MAXIMA I and WMAP, have been reported in Refs. 12 and 42.

\section{ACKNOWLEDGMENTS}

Two of the authors (J.H.P.W. and A.H.J.) acknowledge support from NASA LTSA Grant No. NAG5-6552 and NSF KDI Grant No. 9872979. Another author (P.G.F.) acknowledges support from the RS. Two of the authors (B.R. and C.D.W.) acknowledge support from NASA GSRP Grant Nos. S00-GSRP-032 and S00-GSRP-031. Two of the authors (M.E.A. and R.S.) acknowledge support from NASA Grant No. NRA-00-01-AISR-004. MAXIMA is supported by NASA Grant Nos. NAG5-3941 and NAG5-4454 and by the NSF through the Center for Particle Astrophysics at UC Berkeley, NSF Cooperative Agreement No. AST-9120005. Computing resources were provided by the National Energy Research Scientific Computing Center, which is supported by the Office of Science of the U.S. Department of Energy under Contract No. DE-AC03-76SF00098, and by the University of Minnesota Supercomputing Institute in Minneapolis, Minnesota. MAXIMA field and flight support was provided by the National Scientific Balloon Facility. The MAXIMA team would like to thank P. Timbie for the use a Gunn oscillator and P. Mauskopf for providing electronic readout units. The authors also thank S. Young for his help in preparing figures for this manuscript.

\footnotetext{
${ }^{1}$ S. Hanany et al., Astrophys. J. Lett. 545, L5 (2000).

${ }^{2}$ A. T. Lee et al., Astrophys. J. Lett. 561, L1 (2001).

${ }^{3}$ R. Stompor et al., Astrophys. J. Lett. 561, L7 (2001).

${ }^{4}$ M. E. Abroe et al., Mon. Not. R. Astron. Soc. 334, 11 (2002).

${ }^{5}$ A. Balbi et al., Astrophys. J. Lett. 545, L1 (2000).

${ }^{6}$ L. Cayón, F. Argüeso, E. Martínez-González, and J. L. Sanz, Mon. Not. R. Astron. Soc. 344, 917 (2003).
}

${ }^{7}$ A. H. Jaffe et al., Phys. Rev. Lett. 86, 3475 (2001).

${ }^{8}$ J. L. Sievers et al., Astrophys. J. 591, 599 (2003).

${ }^{9}$ J. A. Rubiño-Martin et al., Mon. Not. R. Astron. Soc. 341, 1084 (2003).

${ }^{10}$ J. H. Goldstein et al., Astrophys. J. 599, 773 (2003).

${ }^{11}$ D. N. Spergel et al., Astrophys. J., Suppl. Ser. 148, 175 (2003).

${ }^{12}$ M. E. Abroe et al., Astrophys. J. 605, 607 (2004).

${ }^{13}$ B. Rabii, Ph.D. thesis, University of California, Berkeley, 2002.

${ }^{14}$ C. D. Winant, Ph.D. thesis, University of California, Berkeley, 2003.

${ }^{15}$ X. Wang, M. Tegmark, B. Jain, and M. Zaldarriaga, Phys. Rev. D 68, 123001 (2003)

${ }^{16}$ J. H. P. Wu et al., Astrophys. J., Suppl. Ser. 132, 1 (2001).

${ }^{17}$ A. H. Jaffe et al., Astrophys. J. 615, 55 (2004).

${ }^{18}$ R. Stompor et al., Phys. Rev. D 65, 022003 (2002).

${ }^{19}$ J. H. P. Wu et al., Phys. Rev. Lett. 87, 251303 (2001).

${ }^{20}$ M. G. Santos et al., Phys. Rev. Lett. 88, 241302 (2002).

${ }^{21}$ M. G. Santos et al., Mon. Not. R. Astron. Soc. 341, 623 (2003).

${ }^{22}$ B. R. Johnson et al., New Astron. Rev. 47, 1067 (2003).

${ }^{23}$ A. H. Jaffe et al., in Microwave Reviews, edited by A. de Oliveira-Costa and M. Tegmark (ASP, San Francisco, 1999), p. 367.

${ }^{24}$ E. Gawiser and G. F. Smoot, Astrophys. J. Lett. 480, L1 (1997).

${ }^{25}$ F. R. Bouchet and R. Gispert, New Astron. 4, 443 (1999).

${ }^{26}$ J. J. Bock, A. E. Lange, M. K. Parikh, and M. L. Fischer, Appl. Opt. 34, 4812 (1995).

${ }^{27}$ J. R. Pardo, J. Cernicharo, and E. Serabyn, IEEE Trans. Antennas Propag. 49, 1683 (2001).

${ }^{28}$ J. J. Bock, Ph.D. thesis, University of California, Berkeley, 1994.

${ }^{29}$ M. Halpern, H. P. Gush, E. Wishnow, and V. de Cosmo, Appl. Opt. 25, 565 (1986).

${ }^{30}$ M. J. Griffin, J. J. Bock, and W. Gear, Appl. Opt. 41, 6543 (2002).

${ }^{31}$ A. D. Olver, P. J. B. Clarricoats, A. A. Kishk, and L. Shafai, Microwave Horns and Feeds (IEEE, New York, 1994).

${ }^{32}$ W. T. Welford and R. Winston, The Optics of Nonimaging Concentrators: Light and Solar Energy (Academic, New York, 1978).

${ }^{33}$ C. Lee, Ph.D. thesis, Queen Mary and Westfield College, London, 1997.

${ }^{34}$ P. Richards, in Spectroscopic Techniques for Far Infrared, Submillimetre and Millimetre Waves, edited by D. Martin (North Holland, Amsterdam, 1967), pp. 33-36.

${ }^{35}$ Handbook of Mathematical Functions with Formulas, Graphs and Mathematical Tables, edited by M. Abramovitz and I. A. Stegun (Dover, New York, 1972).

${ }^{36}$ M. Galeazzi and D. McCammon, J. Appl. Phys. 93, 4856 (2003).

${ }^{37}$ P. L. Richards, J. Appl. Phys. 76, 1 (1994).

${ }^{38}$ S. Hanany, A. Jaffe, and E. Scannanpieco, Mon. Not. R. Astron. Soc. 299, 653 (1998).

${ }^{39}$ E. E. Haller, Third International Conference on Infrared Physics, Zurich, Switzerland, July 23-27, 1984.

${ }^{40}$ E. E. Haller, N. P. Palaio, M. Rodder, W. L. Hansen, and E. Kreysa, in Neutron Transmutation Doping of Semiconductor Materials, edited by R. D. Larrabee (Plenum, New York 1984), p. 21.

${ }^{41}$ J. J. Bock, D. Chen, P. D. Mauskopf, and A. E. Lange, Space Sci. Rev. 74, 229 (1995).

${ }^{42}$ R. Stompor et al., C. R. Phys. 4, 841 (2003). 
${ }^{43}$ P. Timbie, G. Bernstein, and P. Richards, Cryogenics 30, 271 (1990).

${ }^{44}$ C. Hagmann and P. Richards, Cryogenics 35, 303 (1995).

${ }^{45}$ G. F. Smoot et al., Astrophys. J. Lett. 371, L1 (1991).

${ }^{46}$ D. J. Schlegel, D. P. Finkbeiner, and M. Davis, Astrophys. J. 500, 525 (1998).

${ }^{47}$ A. B. Goldin et al., Astrophys. J. Lett. 488, L161 (1997).
${ }^{48}$ E. L. Wright and S. Odenwald, Bull. Am. Astron. Soc. 12, 456 (1980).

${ }^{49}$ D. J. Rudy, Ph.D. thesis, California Institute of Technology, 1987.

${ }^{50}$ P. L. Richards, S. M. Grannan, and M. K. Hase, ESA SP-388: Submillimeter and Far-Infrared Space Instrumentation (ESA, Netherlands, 1996), Vol. 388, p. 143.

${ }^{51}$ P. de Bernardis et al., Nature (London) 404, 955 (2000). 